\title{
XXX. Die thermische Deformation der krystallisirten Sulfate von Kalium, Rubidium und Cäsium.
}

\author{
Von
}

A. E. Tutton in Oxford.

(Hierzu 1 Textfigur.)

In einer früheren Mittheilung (diese Zeitschrift, 30, 529) hat Verfasser ein Interferenzdilatometer beschrieben, durch dessen Gebrauch, wegen der Einführung einer Compensation für die Ausdehnung des Interferenzapparates aus Platin-Iridium vermittelst einer Scheibe von Aluminium, die empfindliche Methode von Fizeau gleich genau bei der Bestimmung der Ausdehnung solcher festen Substanzen wird, insbesondere Krystalle, welche man nicht in relativ grossen ( $1 \mathrm{~cm}$ dicken) Blöcken erhalten kann. Die Methode wird besonders anwendbar bei jenen Substanzen, die Krystalle zahlreicher künstlich dargestellter Salze und anderer Präparate eingeschlossen, deren geschliffene Oberflächen eine Politur gleich derjenigen von Glas nicht annehmen. Der Verfasser musste eine solche Methode ersinnen, um im Stande zu sein, seine Untersuchungen über die Bezichungen zwischen den morphologischen und physikalischen Eigenschaften der Krystalle isomorpher Salzreihen auf der einen Seite und ihrer chemischen Zusammensetzung auf der anderen $\left.{ }^{1}\right)$ auf die thermischen Deformationen jener Salze auszudehnen. Es ist ganz unmöglich, eine hinreichende Politur der geschliffenen Oberflächen des hierzu erforderlichen Krystallblockes dieser Salze zu erhalten. Daher muss man eine Deckplatte einführen, deren Oberfl̈̈che die unentbehrliche, gut reflectirende Politur besitzt. Aus allen möglichen Substanzen wurde das Aluminium ausgewählt, hauptsächlich wegen seiner hohen Ausdehnung (2,6 mal so gross wie diejenige von Platin-Iridium), aber auch weil seine nicht allzugrosse Politur eine solche ist, welche eine Reflexion liefert, die beinahe genau gleich derjenigen von der unteren Fläche des gläsernen auf den Platin-Iridiumschrauben liegenden Deckkeils ist. Die hohe Ausdehnung erlaubt,

1) Diese Zeitschr. 1893, 21, 491; 1894, 24, 1; 1896, 27, 113, 252 und 266; 1897, 29, 63. 
dass man mit einer etwas dicken Scheibe aus Aluminium die Ausdehnung der Platin-Iridiumschrauben mehr oder weniger compensiren kann, was den grossen Vortheil bietet, dass die Zahl der an der Bezugsmarke vorübergehenden Interferenzstreifen bedeutend erhöht wird, und zwar wird dadurch dic der Ausdehnung des Objects selbst entsprechende Zahl, und folglich der Einfluss des mikrometrischen Einstellungsfehlers, ein kleiner Bruchtheil cines Streifens, vermindert. Die günstige Politur des Aluminiums gestattet, dass die dunkeln Streifen nicht durch einen Ueberschuss an von der Deckplatte reflectirtem Lichte geschwächt werden.

Was die Anwendung der Compensationsmethode in möglichen Fällen von Substanzen, welche eine gute Politur annehmen, welche man aber nicht in centimetergrossen Stücken erhalten kann, betrifft, so kann Verfasser nicht aus seiner Erfahrung berichten. Denn die sämmtlichen, vom Verfasser untersuchten Substanzen, welche nicht in centimeterdicken Stücken darstellbar sind, sind Salze, welche eine hinreichende Politur nicht annehmen werden, und welche folglich eine Deckplatte haben müssen. Die Compensationsmethode wurde für solche Fälle beabsichtigt. Verfasser hat sich aber genügende Erfahrung mit der gewöhnlichen Fizeau'schen Methode, was die Anordnung des Interferenzdreifusses und seines Inhaltes betriff, verschafft bei der Bestimmung der Ausdehnung von Aluminium (s. diese Zeitschr. 30, 562) und bei èner kürzlich vollendeten Untersuchung über die Ausdehnung von absolut reinem Nickel und Kobalt (s. S. 384); und die Uebereinstimmung der Einzelbestimmungen dieser Untersuchungen und derjenigen der jetzt mitgetheilten und mit Hülfe der Compensationsmethode ausgeführten Untersuchungen von nichtpolirbaren Salzen, ist von genau derselben hohen Ordnung. Anspruch an eine höhere Genauigkeit für die Compensationsmethode wird gar nicht gemacht. Nach aller Wahrscheinlichkeit sogar, wenn ein Fall eines polirbaren, aber nicht in $5-10 \mathrm{~mm}$ dicken Stücken erhaltbaren Materials für die Untersuchung vorlag, möchte Verfasser die gewöhnliche Fizeau'sche Anordnung vorziehen; denn, obgleich die Compensationsinethode den Streifeneinstellungsfehler vermindert, ist sie doch natürlich complicirter und wird die Einführung eines dritten Factors, die Deckplatte, von der Möglichkeit neu hinzutretender Fehler begleitet, namentlich der Werthe, welche von der Genauigkeit der Bestimmung der Ausdehnung des Deckplattenmaterials abhängen. Solche Möglichkeit von Fehlern aber ist äusserst gering, weil die zahlreichen einzelnen Bestimmungen für Aluminium so übereinstimmend waren (maximale Differenz 0,2 Procent), dass der angewandte Mittelwerth unzweifelhaft sehr genau ist.

In den vorliegenden Fällen künstlicher Salze musste die Compensationsmethode nothwendigerweise angewandt werden; die Resultate sind aber, wie nachher gezeigt werden wird, in sehr zufriedenstellender Weise übereinstimmend. Das reine Aluminium der Compensatoren hat sich nach zwei- 
jährigem Gebrauche ganz unverändert erwiesen, und eine Wiederbestimmung seiner Ausdehnung hat genau dasselbe Resultat geliefert. Ausserdem ist bei einer der Beobachtungen. die Correctur für den Mangel vollkommener Compensation direct bestimmt worden, indem der Compensator ohne Object allein auf die Spitzen des Dreifusstisches aufgelegt wurde; die Beleuchtung und Erzeugung der Słreifen sind im grünen Quecksilberlichte bei grossem Gangunterschiede (grosse Entfernung der reflectirenden Oberflächen) ausgeführt worden. Die so bestimmte Correctur war so nahe identisch mit derjenigen, welche aus der Kenntniss der vorher vom Verfasser bestimmten Ausdehnungscoëfficienten des Platin-Iridiums und des Aluminiums berechnet wurde, dass es für ganz unnöthig angenommen wurde, dieses nach jedem Versuche auszuführen.

Aus allen den vom Verfasser untersuchten Salzreihen, Sulfaten, Doppelsulfaten und Selenaten, welche Kalium, Rubidium und Cäsium enthalten, sind die normalen Sulfate dieser drei Metalle allein bequem für eine Untersuchung über ihre thermische Deformation. Die Doppelsulfate sind nicht passend wegen der Leichtigkeit, mit welcher sie im Allgemeinen ihr Krystallwasser verlieren, wenn man ihre Temperatur erhöht, und dasselbe gilt auch bei den Doppelselenaten, deren Untersuchung mit Rücksicht auf ihre anderen morphologischen und physikalischen Eigenschaften jetzt im Gange ist. Die einfachen Selenate stellen grössere Schwierigkeiten wegen ihrer sehr hygroskopischen Natur entgegen, welche so hervortretend im Falle des Cïsiumselenates ist, zufolge des vom Verfasser (diese Zeitschr. 29, 67) angedeuteten progressiven Fortschrittes der Löslichkeit der drei Salze nach den Atomgewichten der Metalle, dass dieses Salz ein lebhaftes Exsiccationsmittel bildet. Das normale Sulfat von Kalium ist ganz frei von diesem Nachtheile, und sogar ist es eines der am wenigsten löslichen Salze unter denjenigen, welche gewöhnlich in Wasser lösliche Salze genannt werden; sein Löslichkeitsgrad ist nur 10\%. Schwefelsaures Rubidium ist so gering hygroskopisch (Löslichkeit 44\%), dass es keine Schwierigkeit bereitet. Schwefelsaures Cäsium aber ist entschieden hygroskopisch; seine Löslichkeit ist $163 \mathrm{~g}$ in $100 \mathrm{ccm}$ Wasser. Obgleich diese Eigenschaft nicht so ausgeprägt ist, wie im Falle des analogen Selenates, von welchem die Löslichkeit $245 \mathrm{~g}$ in $100 \mathrm{ccm}$ Wasser beträgt, so ist sie doch hinreichend, um den Gebrauch des Salzes für den fraglichen Zweck bei feuchtem Wetter ganz unmöglich zu machen. Dessenungeachtet ist diese Schwierigkeit im Falle des schwefelsauren Cäsiums überwunden worden durch die Benutzung der günstigsten Tage des letzten, ausgezeichnet trocknen Sommers und auch einiger weniger trocknen frostigen Tagen des Winters, neben dem Hülfsmittel der Benutzung der inneren Kammer des Luftbades des Dilatometers als Exsiccator, indem man darin ein mit Schwefelsäure versehenes Gefäss bis zum Augenblicke des Anfanges der Beobachtungen stellt. 


\section{Darstellung der Krystalle.}

Die grösste Schwierigkeit lag darin, Krystalle des gewöhnlichsten der Salze, des schwefelsauren Kaliums, von hinreichender Dicke längs aller drei Axenrichtungen zu erhalten. Burch langsames Verdunsten kalt gesättigter Lösungen über Schwefelsäure im Vacuum wird es müglich, hinreichende Zeit und Lüsungsmenge angenommen, vortreffliche Krystalle der löslicheren Sulfate von Rubidium und Cäsium darzustellen, welche die erforderliche Grösse besitzen, um Blöcke von 5-10 mm Dicke in jeder der drei Axenrichtungen zu liefern. Aber im Falle des weniger lüslichen Kaliumsalzes sind die Krystalle gewöhnlich klein. Ausserdem, wenn sie ausnahmsweise grösser sind, sind sie entweder pseudohexagonale Drillinge oder andere Zwillingsformen, ganz unbrauchbar für unseren Zweck wegen der ungleichen Ausdehnung der durcheinander gewachsenen Theile, oder sie sind langprismatisch nach der Richtung der $\Lambda$ xe $a$ und zu schmal für den Gebrauch längs der beiden anderen $\Lambda$ xenrichtungen $b$ und $e$, und besonders längs $b$. Es ist sehr schwer gefunden worden, gut ausgebildete, aus einer grossen Menge abkühlender Lösung abgesetzte Prismen bis zur. gewünschten Grösse in kalt gesättigten Lösungen über Schwefelsäure im Vacuum weiterwachsen $\mathrm{zu}$ machen. Nachdem sie eine Dicke von circa $4 \mathrm{~mm}$ erreicht hatten, kam es immer vor, dass, anstatt weiter zu wachsen, frische Krystalle auf ihnen sich abzusetzen anfingen.

Nach mehrere Monate langen fruchtlosen Versuchen appellirte Verfasser an verschiedene chemische Firmen, unter anderen Merck in Darmstadt, in der Hoffnung, dass bei dem Gebrauche von viel grösseren Mengen des Materials grössere Krystalle erhalten werden könnten. Endlich ist es Herrn Hopkin und Williams gelungen, sieben Prismen von ausnahmsweiser Dicke zu erhalten, und diese haben, nach ein wenig weiterem Wachsthum in einer kalt gesättigten Lösung im Vacuum, durchsichtige Blöcke von 6,5 bis $9,6 \mathrm{~mm}$ Dicke längs der Richtung der Axe $c$ und 5,1 bis $5,9 \mathrm{~mm}$ längs $b$ geliefert.

\section{Herstellung der mit parallelen Flächen versehenen Krystallblöcke.}

Die Herstellung der Krystallblöcke, von welchen jeder mit einem Paare genau ebener und paralleler Flächen versehen wurde, genau senkrecht zu der betreffenden krystallographischen Axe, längs welcher die lineare Ausdehnung oder Zusammenziehung zu bestimmen war, ist vermittelst des neuen, in der nachfolgenden Mittheilung beschriebenen Schneid- und Schleifgoniometers ausgeführt worden. Es wurden Krystalle ausgewählt, welche frei von Spuren von Trübung, von Spalten und von Verzerrung waren. Nach ihrem Wegnehmen aus der Mutterlauge wurden die Krystalle getrocknet, und erst in einem Exsiccator mehrere Tage vor dem Gebrauch 
aufhewahrt. Mil zwei Ausnahmen wurde jeder ausgewïhlte Krystall nur für zwei, an zwei nacheinander folgenden 'lagen vorgenommenen Bestimmungen der linearen thermischen Ausdehnung oder Zusammenziehung längs einer besonderen Axenrichtung angewandt. Im Ganzen wurden 29 verschiedene Krystalle gebraucht, 11 von schwefelsaurem Kalium, 8 von schwefelsaurem Rubidium und 10 von schwefelsaurem Cäsium. Eine der Ausnahmen war ein besonders schöner Krystall des Rubidiumsalzes, welcher so lang in einer Axenrichtung war, dass er in zwei Ï̈̈lften geschnitten wurde, und diese Theile waren noch so gross, dass sie für Bestimmungen längs zwei verschiedener $\Lambda$ xenrichtungen angewandt wurden. Die andere Ausnahme war ein Krystall des schwefelsauren Cäsiums, welcher in ausgezeichnet trocknem Wetter zu einem rechtwinkeligen Blocke geschnitten und geschliffen wurde, für nacheinander folgende Bestimmungen längs aller drei Axenrichtungen, um ein Beispiel darzustellen, bei welchem alle drei linearen Werthe, und aus diesen der Werth für die kubische Ausdehnung, aus einem und demselben Krystalle erhalten sein sollten; eine Vergleichung der erhaltenen kubischen Ausdehnung mit derjenigen, welche durch Berechnung aus den Bestimmungen der drei von drei verschiedenen Krystallen gelieferten linearen Werthe erhalten wurde, war sehr zufriedenstellend.

Die Orientirung der verschiedenen Krystallflächen und das Identificiren der Axenrichtungen wurden stets durch goniometrische Messungen und Untersuchung der Interferenzbilder im convergenten polarisirten Lichte bestätigt. Eintauchen in Benzol hilft beträchtlich bei der letzteren Untersuchung, da der Brechungsexponent jener Flüssigkeit beinahe das Mittel der Exponenten der drei Salze ist; die Bilder sind folglich sehr klar und der scheinbare Axenwinkel ist beinahe gleich dem wahren. Oel darf man nicht anwenden, weil es in etwa vorhandene kleine Höhlungen hineingeht und während der thermischen Beobachtungen in sehr kleinen Tröpfchen, welche die Interferenzstreifen durch Aufhebung des Compensators stören, wieder abläuft.

Wegen einer ähnlichen Ursache darf man kein Oel beim Schleifen der Flächen anwenden; es wurde anstatt dessen das sehr flüchtige Benzol gebraucht. Natürlich aber hat man grüssere Mengen $\mathrm{zu}$ verwenden; folglich wurde ein Trichter über der Schneid- oder Schleifscheibe so angebracht, dass die Tropfen hinreichend schnell nacheinander auf die Schlifffläche fielen, um diese genügend nass zu erhalten.

Der Krystall wurde in einem der Griffhalter (s. die folgende Mittheilung) mit der Axenrichtung, längs welcher man die lineare thermische Deformation zu bestimmen wünschte, approximativ vertical montirt und daher senkrecht zu den Schneid- und Schleifscheiben. Die Justirung der Axenrichtung wurde dann absolut gemacht, jndem man die dazu parallele Flächenzone goniometrisch justirte. Wenn der Krystall am Ende so ausgebildet 
war, dlass vicles Schleifen nothwendig war, un die erwünschte Oberfläche herzustellen, so wurde zuerst die Schneidscheibe gebraucht, um das untere Lnde in einer solchen Ilöhe abzuschneiden, dass die gewünschte Oberfläche mit minimalem Verluste des Materials geliefert wurde. Das Schnejden solcher künstlicher Salzkrystalle gelingt in sehr zufriedenstellender Weise mit dem Schneideapparat des Verfassers, und zwar ist noch nicht ein Krystall bis jetzt zerbrochen worden. Wenn kein Schneiden nothwendig ist, führt man das vorläufige Anschleifen der Oberflïche mit Hülfe der Schmirgeltuchscheibe aus; zuerst mit dem Krystallhalter und die Scheibe von dem Instrument getrennt und der erstere in der Fand gehalten, und nachher, nachdem eine Approximation der erwünschten Oberlläche erreicht wird, mit dem Krystall und seinem llalter und der Scheibe in ihrer Stellung cingerichtet. Endlich, nach Bestätigung der Justirung, welche wegen der Art der Befestigung des Ilalters auf dem Justirungsapparate gewöhnlich unverändert geblieben ist, wurde die Oberfläche fein geschliffen vermittelst einer der Mattglasscheiben. Sie wurde endlich polirt mit der gläsernen Polirscheibe. Viel Zeit wurde bei der letzten Operation nicht aufgewendet, und gar keine in dem Falle des hygroskopischen Cüsiumsalzes; denn die Compensationsscheibe aus Aluminium liefert, wie schon erklärt, die reflectirende Oberfläche, und die beste mögliche Feinpolitur des Kaliumsalzes ist nicht hinreichend, um ohne Deckscheibe zu arbeiten. Es war daher nur nüthig, den Block fertig zu machen, indem man ihn mit einer ähnlichen parallelen Oberfläche versah. Der Krystallblock wurde alsdann mit Benzol gewaschen und in den Exsiccator gestellt. Die Dicke der Blöcke variirte von $4,8 \mathrm{~mm}$ (ausnahmsweise dünn) bis $10,7 \mathrm{~mm}$.

In einigen wenigen Fällen wurden die Ausdehnungsbeobachtungen durch lierauslaufen von Mutterlaugespuren zwischen Krystall und Compensator oder Dreifusstisch unmöglich gemacht. In zahlreichen Fällen war die Dreipunktcontactmethode nicht anwendbar, weil die geschliffenen Krystalloberflächen in einer Richtung zu schmal waren, um auf dem dritten Punkte zu liegen, aljer nicht zu schmal für stabiles Gleichgewicht des Krystalls und Compensators. Eine Vergleichung der Resultate für dieselbe Richtung bei beiden Methoden zeigt keine Jemerkbare Differenz. Die Oberflächen waren immer absolut rein und frei von Staubpartikeln und so absolut eben, dass gar kein Rollen, durch geringe Convexität hervorgebracht, je entdeckt worden ist. Die Aufstellung des Krystalls und des Compensators auf dem Ireifuss wurde immer durch Gleitung bewirkt, um irgend eine andernfalls dazwischen kommende Iuftschicht $\mathrm{zu}$ beseitigen. In den Fällen der erwähnten herauslaufenden Mutterlaugespuren wurden die Krystalle nachher langsam auf $105^{\circ}$ in einem Luftbade erhitzt, und bei dieser Temperatur zwülf Stunden erhalten. Alsdann. wurden die Beobachtungen wiederholt, und sic gelangen jedesmál gut. Die Resultate stimmten in zu- 
friedenstellender Weise mit denjenigen überein, welche für dieselbe Richtung desselben Salzes erhalten worden waren in Fällen, bei welchen diese Behandlung nicht nothwendig war.

\section{Die Beobachtungen.}

Die Bestimmungen der thermischen Ausdehnung (Zusammenziehung wurde bei diesen Salzen niemals gefunden) wurden in der Weise ausgeführt, welche sehr kurz für die Krystalle am Ende der Dilatometermittheilung (diese Zeitschr. 30, 566) angedeutet worden ist. Die angewandten Temperaturen sind nicht ganz so hoch gewesen, als bei den Bestimmungen der Ausdehnung von Platin-Iridium und Aluminium. Die höchste Grenze war ca. 96 $6^{\circ}$, damit keine bemerkbare Deformation vorkommen könnte, hervorgebracht durch innere Spannung, weiche durch die Ausdehnung der Mutterlauge in den immer anwesenden sehr geringen Höhlungen verursacht wird.

Damit die Bestimmungen streng vergleichbar wurden, ist so häufig wie möglich dieselbe Aluminiumscheibe gebraucht worden, welche eine Dicke von $5,25 \mathrm{~mm}$ und $1 \mathrm{~cm}$ Durchmesser hat und keine hervorragenden Punkte trug. Wo aber ausnahmsweise die Dreipunktmethode angewandt worden ist, sind andere Resultate mit der $5,25 \mathrm{~mm}-$ Scheibe für dieselbe Richtung desselben Salzes vorhanden, und die beiden Resultatreihen stimmen gut überein. Dieses war zu erwarten, denn die Aluminiumscheiben sind alle aus demselben Gusse geschnitten worden. Die $5,25 \mathrm{~mm}$-Scheibe liefert vortreff liche Interferenzstreifen, und die Streifen von der immer gebrauchten Fläche waren ausserordentlich wenig gekrümmte Theile grosser Kreise, durch kaum bemerkbare Convexität verursacht.' Dieses war ein Vortheil, da es müglich war, dadurch zu bestimmen, ob die Bewegung der Streifen durch Ausdehnung oder Kusammenziehung verursacht war, zufolge deren sie sich entweder von dem Krümmungscentrum weg oder nach ihm hin bewegten.

Die Luftschicht zwischen den zwei reflectirenden Oberflächen war beinahe immer sehr dünn; so wurde es vortheilhaft gefunden, weniger nach genauer Compensation für die Ausdehnung der Schrauben zu streben, als nach Herstellung hellerer Streifen. Denn die Correctur für ungenügende Compensation ist immer bestimmt aus den bekannten Ausdehnungen der Dreifusslegirung und des Aluminiums. Die $5,25 \mathrm{~mm}$ Aluminium entsprechende Schraubenlänge ist.ca. 13,77 mm, welches Raum für einen Krystall von $8,37 \mathrm{~mm}$ Dicke und eine Luftschicht von 0,15 mm lässt. Da die Krystalle gewöhnlich nur ein oder zwei Millimeter von jeder Seite der erwähnten Dicke variirten, war der nicht compensirte Betrag niemals gross.

Dieselben Thermometer sind angewandt worden, welche früher beschrieben worden sind (1. c. 543). Ihre $0^{\circ}-$ und $100^{\circ}-M a r k e$ wurden vorsichtig nach der Vollendung der Beobachtungen wiederbestimmt. Das innere gebogene Thermometer, dessen cylindrisches Gefäss in Berührung 
mit dem Dreifuss war und dessen $\Lambda$ blesungen angenommen wurden, hat sich nur um $0,1^{\circ}$ verändert; die Ablesungen bei $0^{0}$ und $100^{\circ}$ (in Eis und Wasserdampf), nach Gebrauch der Druckcorrection für den letzteren, waren $0,1^{0}$ und resp. $100,1^{\circ}$. Also ist das Intervall unverändert geblieben, und da nur Temperaturdifferenzen bei den Berechnungen der Ausdehnungscoëfficienten angewandt sind, ist gar keine Correction nothwendig.

Der modus operandi erforderte den grösseren Theil von drei Tagen, um eine Doppelreihe von Bestimmungen der linearen Deformation irgend eines Krystalles längs der Richtung, welche senkrecht zu den zwei hergestellten parallelen Oberflächen war, auszuführen. Der Nachmittag des ersten Tages wurde zur Justirung des Krystalles und des ganzen Apparates verwendet, um ein bequemes Feld von Interferenzstreifen herzustellen. Jeder der zwei nachfolgenden Tage wurde mit den Beobachtungen der Stellung und des Durchganges der Streifen für zwei Temperaturintervalle ausgefüllt, und zwar dauerten die Operationen $\mathbf{5 - 7}$ Stunden bei jedem, während welches ganzen Zeitraumes der Verfasser die Streifen ohne Unterbrechung verfolgte.

Die weitere Erfahrung lehrte, dass für Krystalle die Abbe'sche Methode, die Berechnung der Streifenzahl aus anfänglichen und endlichen Beobachtungen der Stellungen der an der Bezugsmarke nächsten Streifen, für Licht von zwei Wellenlängen, im Allgemeinen unbrauchbar ist. Die einzige Gewähr, dass die Bestimmung eine zuverlässige ist, dass keine durch Erderschütterung, Krystallspaltung oder andere Ursachen bedingte Störungen vorgekommen sind, ist allein durch den vorsichtigsten Verfolg und Zählen der Streifen während des ganzen Intervalles geliefert. Dieselben müssen ihre regelmässigen Entfernungen behalten und keine bemerkbare Drehung oder andere Unregelmässigkeit zeigen. Häufig wird eine geringe Spaltung des Krystalles von einer Erweiterung oder Zusammenziehung der Streifen während eines Theiles der Beobachtung und der entgegengesetzten Bewegung während des anderen begleitet, oder sogar mit einer ganzen Umdrehung, und zwar häufig durch das blosse Ueberspringen von mehreren Streifen, so dass die Erscheinung am Ende sehr ähnlich derjenigen am Anfange ist. Eine solche Beobachtung ist natürlich werthlos, aber die Abbe'sche $\mathrm{Me}-$ thode würde dieses nicht entdecken lassen. Also ist die oben beschriebene die einzige zuverlässige Methode, wenn zerbrechliche Substanzen in Untersuchung sind.

Das Zählen der Streifen wurde genau ausgeführt, wie in der Dilatometermittheilung beschrieben worden ist (1. c. S. 554), mit Hülfe des Papierbandzählapparates. Der Inductionsapparat, welcher das mit Wasserstoff versehene Riedel-Geissler'sche Vacuumrohr zum Leuchten bringt, wurde in hinreichend küurzen Zeitintervallen in Thätigkeit gesetzt, um die Beobachtung des Vorübergehens jedes Viertelstreifens zu ermöglichen. Die Uhr wurde

Groth, Zeitschrift f. Krystallogr. XXXI. 
daneben angewandt, um sicher zu sein, dass keine plützliche Veränderung der Schnelligkeit des Streifenvorübergehens vorgekommen ist.

Die angewandten Temperaturgrenzen waren die gewöhnlichen, so niedrig wie müglich gehalten, indem man Morgens früh (7 Uhr) anfing und bis ca. 56 resp. ca. $96^{0}$ ging. Die Bestimmung der diesen beiden Temperaturen entsprechenden Stellungen der zwei nächsten Streifen zur Bezugsmarke (das Centrum eines auf der unteren Seite des gläsernen Deckkeils befindlichen Silberringes) wurde genau so ausgeführt, wie in der Dilatometermittheilung beschrieben worden ist (1. c. S. 553). Das durchaus angewandte monochromatische Licht war rothes Wasserstofflicht, der $C$-Linie des Spectrums entsprechend, von allen anderen Strahlen durch zwei Refractionsprismen getrennt, wie ausführlich in der genannten Mittheilung (S. 536) beschrieben worden ist.

Wenn eine für das Abkühlen hinreichende Zeit nach der zweiten Beobachtungsreihe verflossen war, wurde die Messung der Krystalldicke und der Schraubenlänge ausgeführt mit Hülfe des Dickenmessers (l. c. S. 54.7). Für diesen Zweck wurde die Interferenzkammer vorsichtigst aus dem Bade vermittelst des Triebwerkes herausgehoben und der Dreifuss nebst seinem Tische und dem darauf befindlichen Krystalle und der Aluminiumscheibe, nachdem der das Thermometer verbindende Faden zerschnitten und das Gefäss des Thermometers aus dem Wege gedreht war, aus der Kammer herausgezogen und zum Dickenmesser übertragen, ohne den Inhalt des Dreifusstisches zu stören. Da der Deckkeil zu gross ist, um mit dem Dreifuss durch das Fenster der Kammer zu kommen, so wird er dahinter gelassen, mit dem Silberringe nach oben. Der Dreifuss wurde so auf die gläserne Basisplatte des Dickenmessers aufgestellt, dass das Achatende der Messungsstange genau auf das Centrum der Aluminiumscheibe fallen würde, über welchem der Silberring des Deckkeils während der Beobachtungen gewesen war. Die Höhe der Ebene der Schraubengipfel an diesem Punkte wurde zuerst bestimmt, indem man eine grosse, dem Deckkeil ähnliche Glasscheibe auf die Schrauben auflegte, deren Oberflächen genau eben waren und deren Dicke früher in einer Stellung bestimmt worden war, welche bequem mit einer inneren Luftblase markirt war. Die Scheibe wurde so aufgelegt, dass die Blase über das Aluminiumcentrum kam. Die gemessene Hühe dieser Scheibe, minus der bekannten Scheibendicke, gab die Schraubenhühe. Die Glasscheibe wurde alsdann weggenommen und die Messungsstange sanft auf die Aluminiumscheibe heruntergelassen; alsdann ist die Differenz zwischen dieser Höhe und der Schraubenhöhe die Dicke der Luftschicht. Die Stange wurde wieder gehoben und die Aluminiumscheibe ohne Störung des Krystalles weggenommen; die Stange wurde wieder gesenkt, bis sie auf dem Krystall ruhte; alsdànn wurde eine andere Messung ausgeführt. Die Differenz dieser und der letzteren gab die Dicke des Aluminiums. Eine andere 
Messung ohne den Krystall und mit der Stange in Berührung mit dem Platin-Iridiumtische, gab nach $\Lambda$ bziehen der letzteren die Krystalldicke. Wenn die Dreipunktberührungsmethode gebraucht wurde, wurde die Punkthühe mit der Hülfe einer kleinen Glasscheibe bestimmt, welche im Centrum eine bekannte Dicke besass. Die Schraubenlänge wurde natürlich erhalten nach der angewandten Berührungsmethode, indem man von der Schraubenhöhe die Höhe entweder des Tisches selbst oder der Punkte abzog.

Da die Krystalle orthorhombisch sind, bestand die Aufgabe in der Bestimmung von neun Grössen, nämlich der linearen thermischen Ausdehnungscoëfficienten für jede der drei krystallographischen Axenrichtungen von jedem der drei Salze. Aus diesen Quantitäten kann man bekanntlich die kubischen Ausdehnungscoëfficienten jedes Salzes berechnen; mit anderen Worten, die Volumdifferenz zwischen einer Kugel von Radius 1 und dem daraus durch die Wirkung der Temperaturveränderung erhaltenen Deformationsellipsoide:

Jeder dieser neun linearen Coëfficienten ist wenigstens sechs unabhängige Male bestimmt worden, an drei verschiedenen Krystallen, und fünf davon achtmal an vier Krystallen. Die Gesammtzahl ist 64 Bestimmungen, jede an einem anderen Tage und mit den früher erwähnten 29 verschiedenen Krystallen. Jede Bestimmung lieferte, wie schon vollständig erklärt worden ist (l. c. S. 558), die zwei Constanten $a$ und $b$ des allgemeinen Ausdruckes für irgend eine Temperatur $t$ des Ausdehnungscoëfficienten $a=a+2 b t$. Man hat nur in drei Gleichungen der Form

$$
L_{t}=L_{0}\left(1+a t+b t^{2}\right)
$$

die bestimmten Werthe der drei Temperaturen und der Längen (Dicken) des Krystallblockes bei jenen Temperaturen einzustellen und die drei dadurch erhaltenen Gleichungen für die drei unbekannten Grössen zu lösen, nämlich $L_{0}, a$ und $b$. Die Lüsungen dieser Gleichungen liefern Ausdrücke für die letzteren von der folgenden Form:

$$
a=\frac{\Theta}{L_{0}}, \quad b=\frac{\Phi}{L_{0}} \quad \text { und } \quad L_{0}=L_{t_{1}}-\Theta t_{1}-\Phi_{t_{1}}{ }^{2},
$$

in welchen $\Theta$ und $\Phi$ Glieder sind, welche die Differenzen der Längen $L_{t_{1}}$, $L_{t_{2}}, L_{t_{3}}$ bei den drei Temperaturen $t_{1}, t_{2}, t_{3}$ und die Summen und Differenzen jener Temperaturen enthalten.

Die in den Berechnungen angewandten Ausdrücke für $\Theta$ und $\Phi$ waren:

$$
\begin{aligned}
& \Theta=\frac{\left(t_{1}+t_{3}\right)\left(L_{t_{2}}-L_{t_{1}}\right)}{\left(t_{2}-t_{1}\right)\left(t_{3}-t_{2}\right)}-\frac{\left(t_{1}+t_{2}\right)\left(L_{t_{3}}-L_{t_{1}}\right)}{\left(t_{3}-t_{1}\right)\left(t_{3}-t_{2}\right)}, \\
& \boldsymbol{\Phi}=\frac{L_{t_{3}}-L_{t_{1}}}{\left(t_{3}-t_{1}\right)\left(t_{3}-t_{2}\right)}-\frac{L_{t_{2}}-L_{t_{1}}}{\left(t_{2}-t_{1}\right)\left(t_{3}-t_{2}\right)} .
\end{aligned}
$$

Die Resultate sind in den folgenden Tabellen zusammengestellt. Jed $\epsilon$ Tabelle ist in drei Theile getheilt, Versuchsdaten, berechnete wirkliche Aus- 
dehnungen und berechnete lineare Ausdehnungscoëfficienten. In dem ersten Theile ist $L_{t_{1}}$ die gemessene Dicke des Krystallblockes, $l_{a}$ diejenige der Aluminiumscheibe, $l$ die Schraubenlänge und $d$ die Dicke der Luftschicht. Nach den Temperaturen und barometrischen Drucken kommt $f_{2}$, die dem ersten Intervalle $t_{2}-t_{1}$ entsprechende beobachtete Streifenzahl, hiernach ist die Correction für die Veränderung der Luftrefraction, welche vollständig in der Dilatometermittheilung (S. 556) erklärt worden ist, und dann $f_{2}^{\prime}$, die damit corrigirte Streifenzahl; ähnlich für das grosse Intervall $t_{3}-t_{1}$ folgen die beobachtete Streifenzahl $f_{3}$, seine Correctur, und $f_{3}{ }^{\prime}$ die corrigirte Streifenzahl.

In dem zweiten Theile sind zuerst dic berechneten Dickenänderungen der Luftschicht.gegeben, welche erhalten wurden durch Multipliciren der corrigirten Streifenzahlen mit der Halbwellenlänge 0,0003281 des angewandten $C$-Wasserstofflichtes. Zunächst kommen dann die Schraubenausdehnungen, berechnet mit Hülfe der vom Verfasser bestimmten (1. c. S. 559) Ausdehnungscoëflicienten des Platin-Iridiums aus der Formel:

$$
l\left[10^{-9}\left(8600+4,56 \frac{t_{1}+t_{2}}{2}\right)\right]\left(t_{2}-t_{1}\right)
$$

für das erste Intervall und eine ähnliche für das zweite, wobei $t_{3}$ für $t_{2}$ eingesetzt wird. Nachher folgen die in ähnlicher Weise berechneten Ausdehnungen der Aluminiumscheibe aus den Formeln

$$
l_{a}\left[10^{-9}\left(2204+2,12 \frac{t_{1}+t_{2}}{2}\right)\right]\left(t_{2}-t_{1}\right)
$$

und dieselbe, wenn $t_{2}$ durch $t_{3}$ ersetzt ist. Für die Bestimmung des dabei gebrauchten Ausdehnungscoëfficienten des Aluminiums siehe die Dilatometermittheilung S. 562 .

In den nächsten zwei Columnen kommen die Differenzen der Ausdehnungen der Schrauben und der Aluminiumscheibe, d. h. die Correctionen für Nicht-Compensation. Die Correction ist offenbar positiv, wenn ein sich ausdehnender Krystall vorliegt, da die Schrauben sich am meisten ausdehnen, und negativ, wenn die Aluminiumscheibe sich in grösserem Betrage ausdebnt. Die in den letzten zwei Columnen gegebenen Werthe für die wirklichen Ausdehnungen des Krystalles werden durch Anwendung der Correctionen auf die scheinbaren beobachteten Ausdehnungen $f_{2}^{\prime} \lambda / 2$ und $f_{3}{ }^{\prime \lambda} / 2$ erhalten.

In dem dritten Theile der Tabelle sind die berechneten Werthe von $\Theta, \Phi$ und $L_{0}$ gegeben und darnach auch von den zwei gesuchten Constanten des Ausdehnungscoëfficienten $a$, dem Coëfficienten bei $0^{0}$, und $b$, dem halben Increment des Coëfficienten pro Temperaturgrad. Endlich ist der Werth des Ausdehnungscoëfficienten $\alpha$ für $50^{\circ}$ gegeben, aus der Formel $\alpha=a+2 b t$ berechnet für $t=50^{\circ}$. Diese Temperatur war gewählt, weil sie näher dem Mittel der Temperaturgrenzen war als $40^{\circ}$, die von Fizeau gewühnlich gebrauchte mittlere Temperatur. 

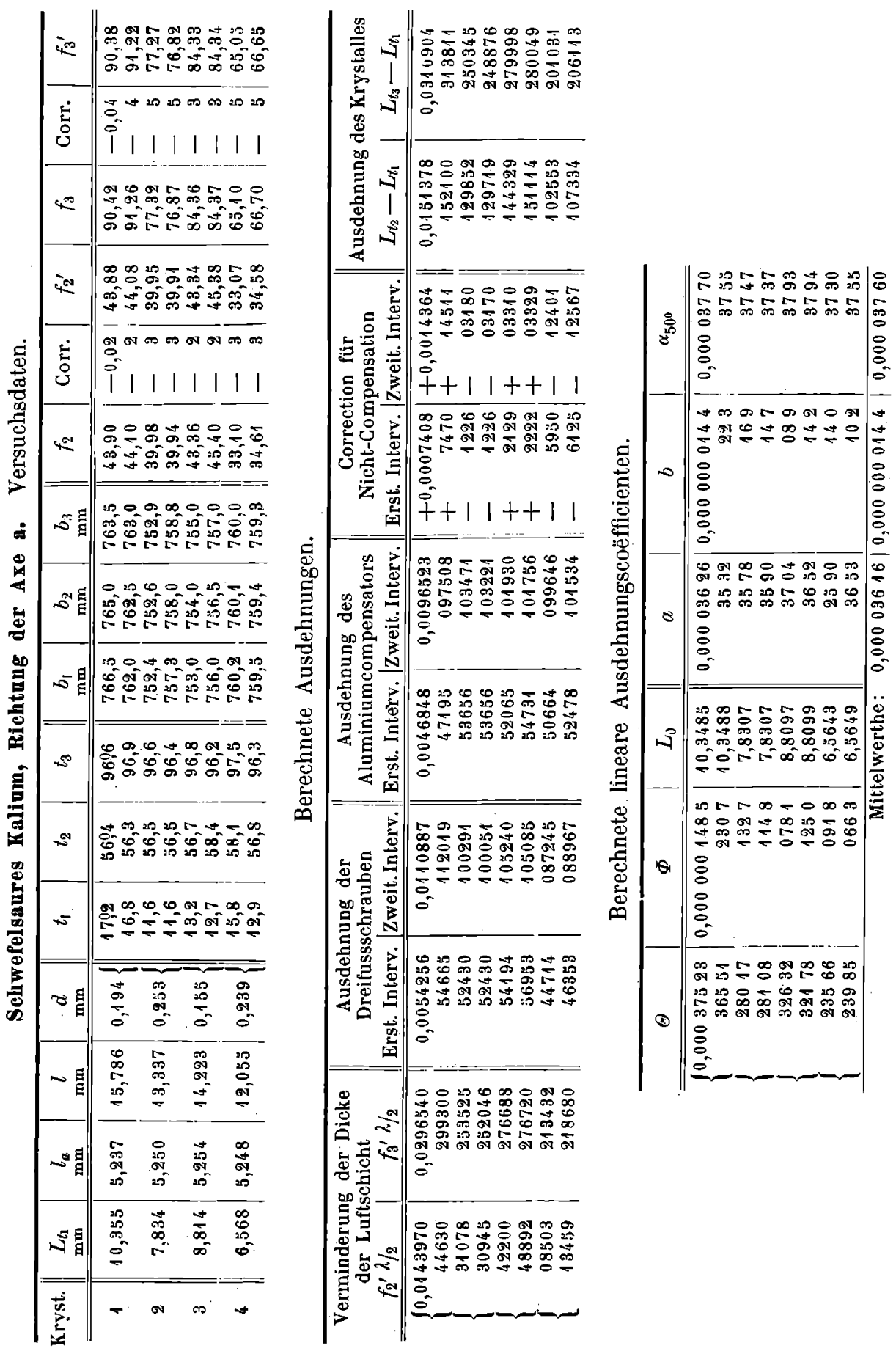
A. E. Tutton.

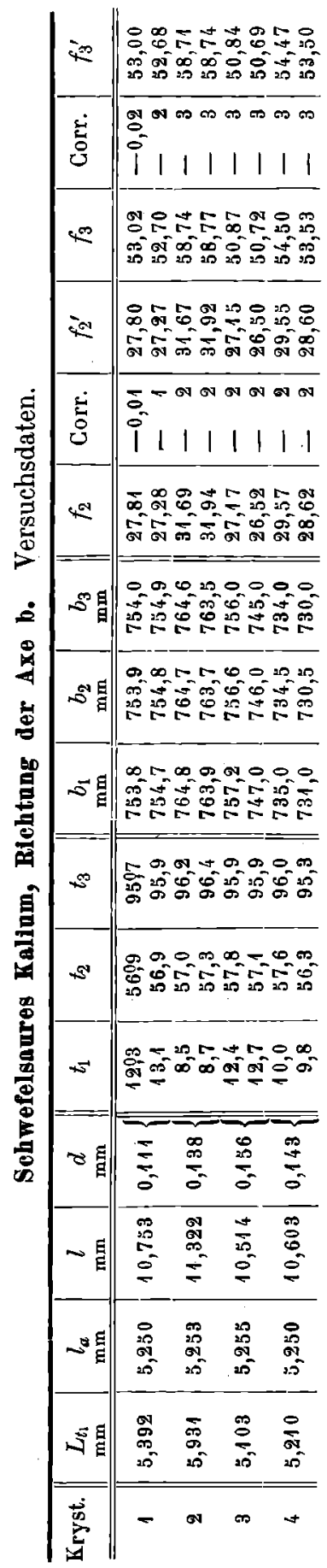
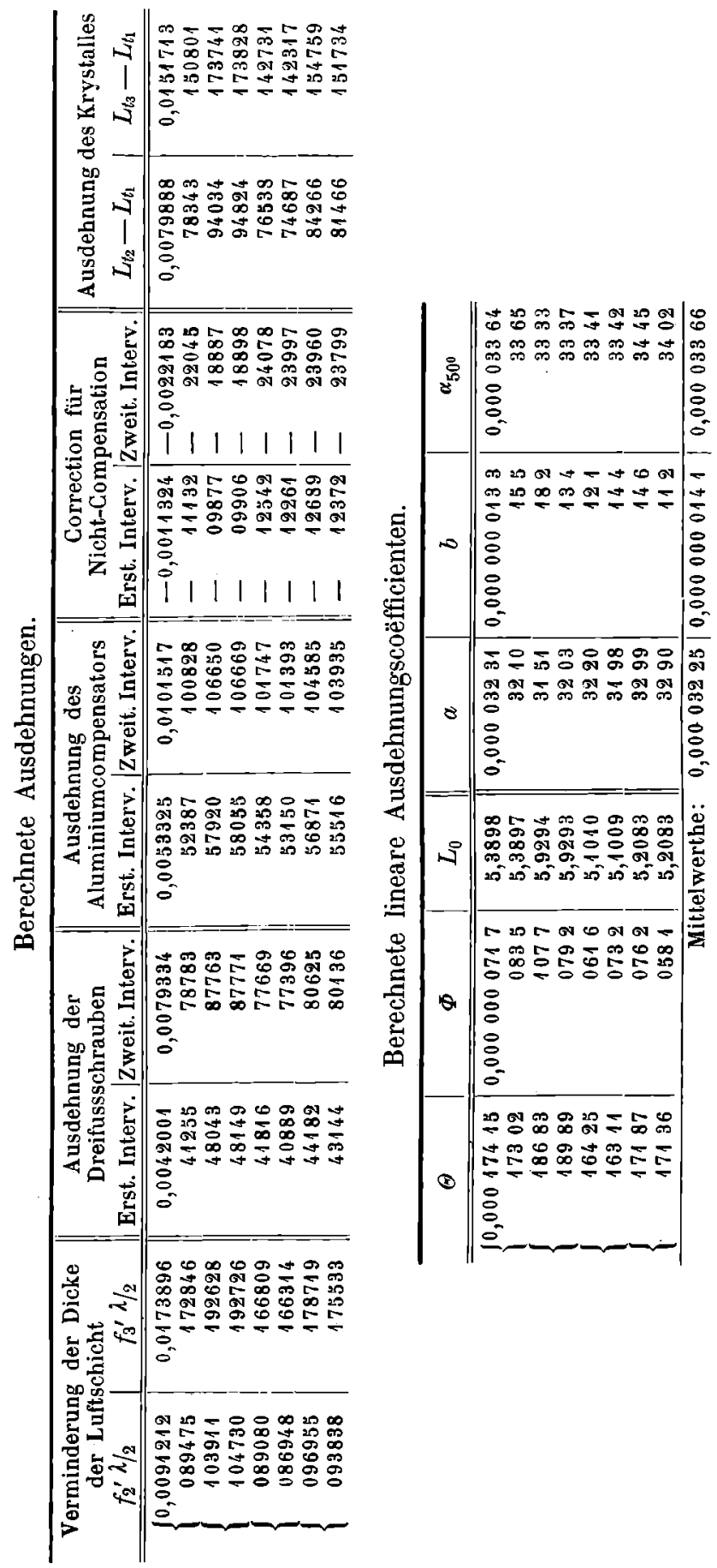

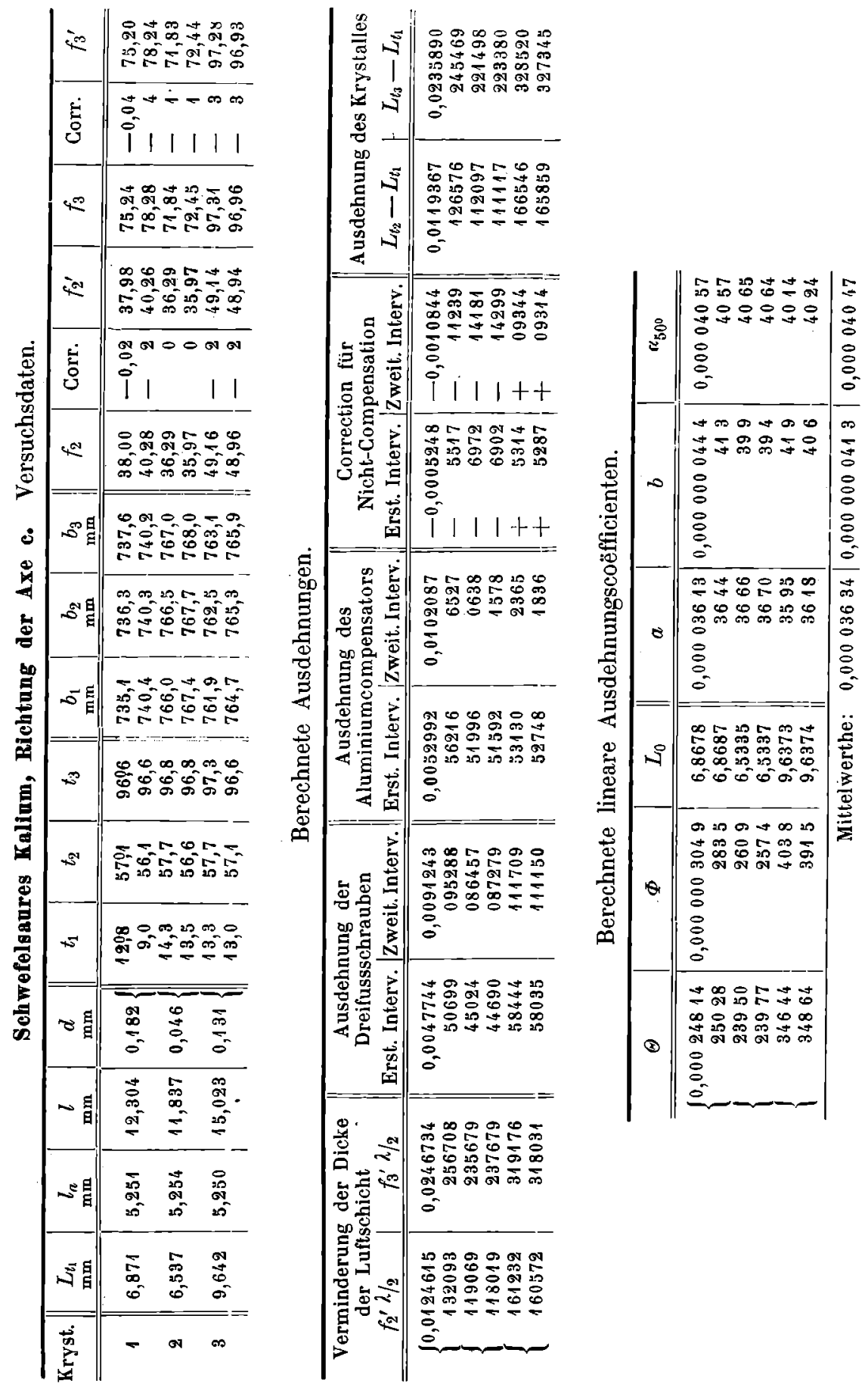
A. E. Tutton.
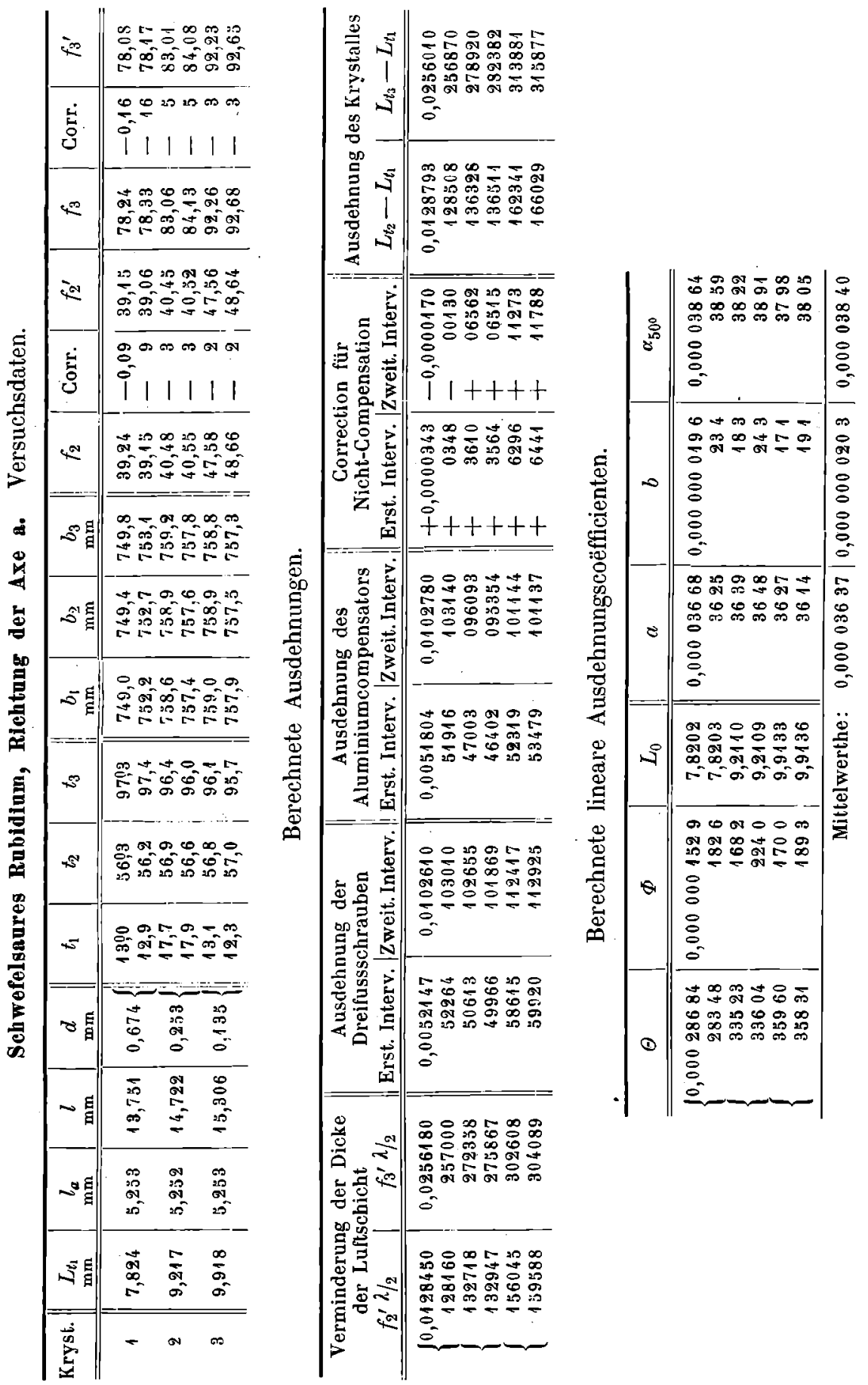

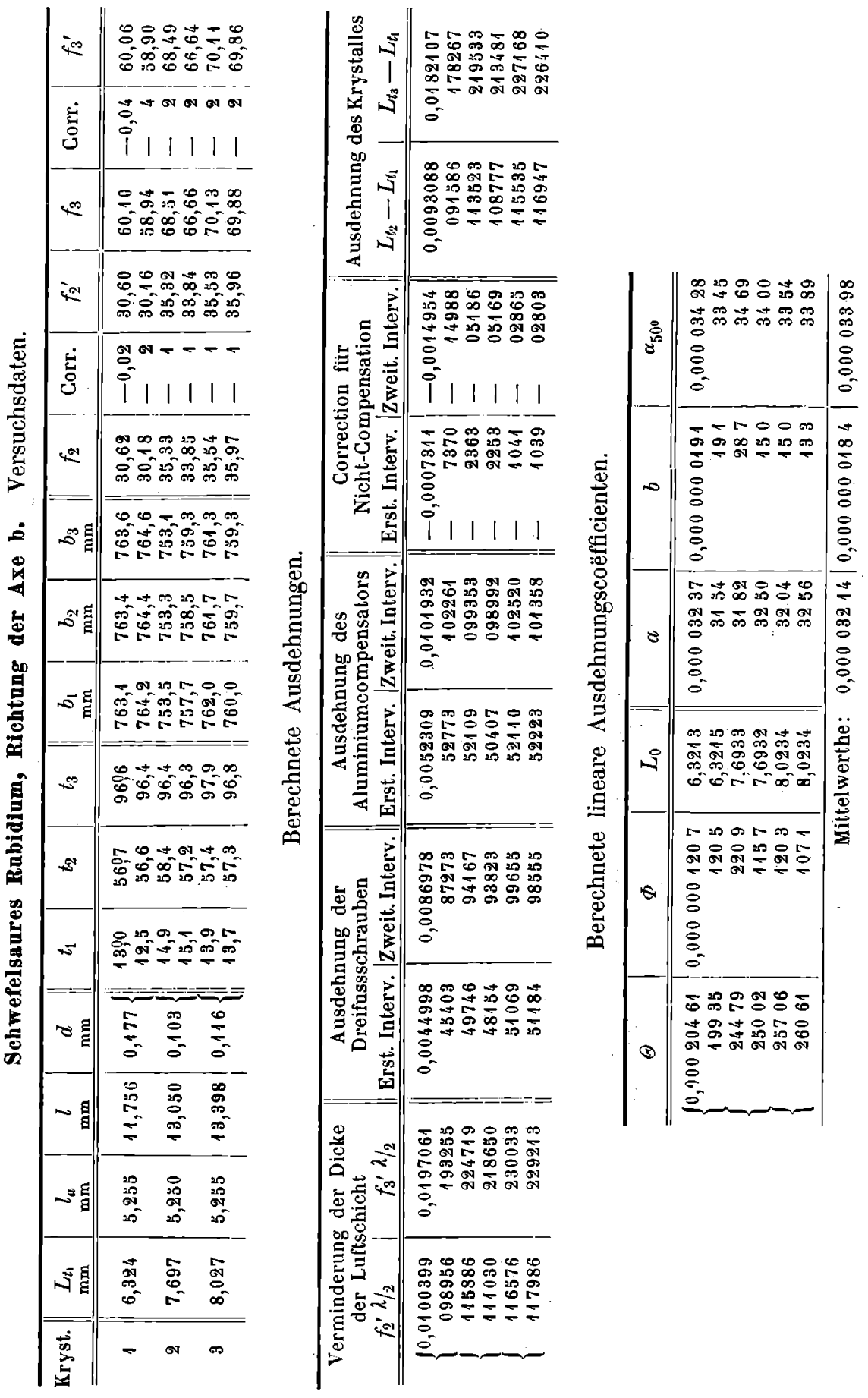

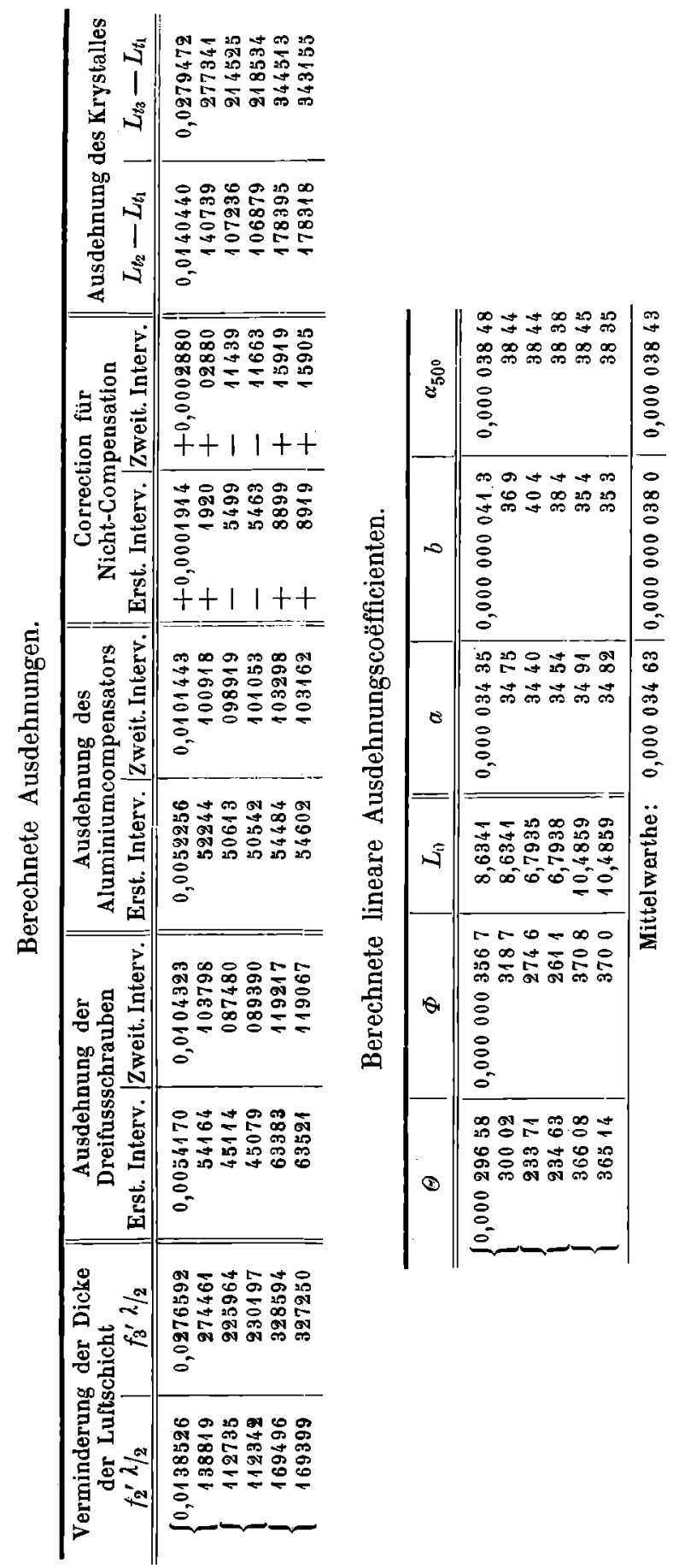


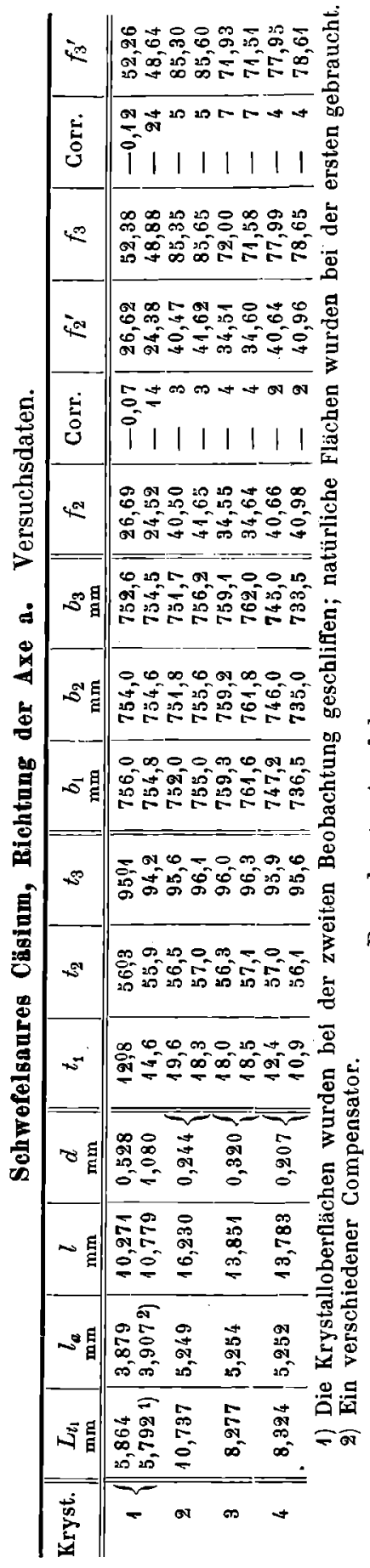

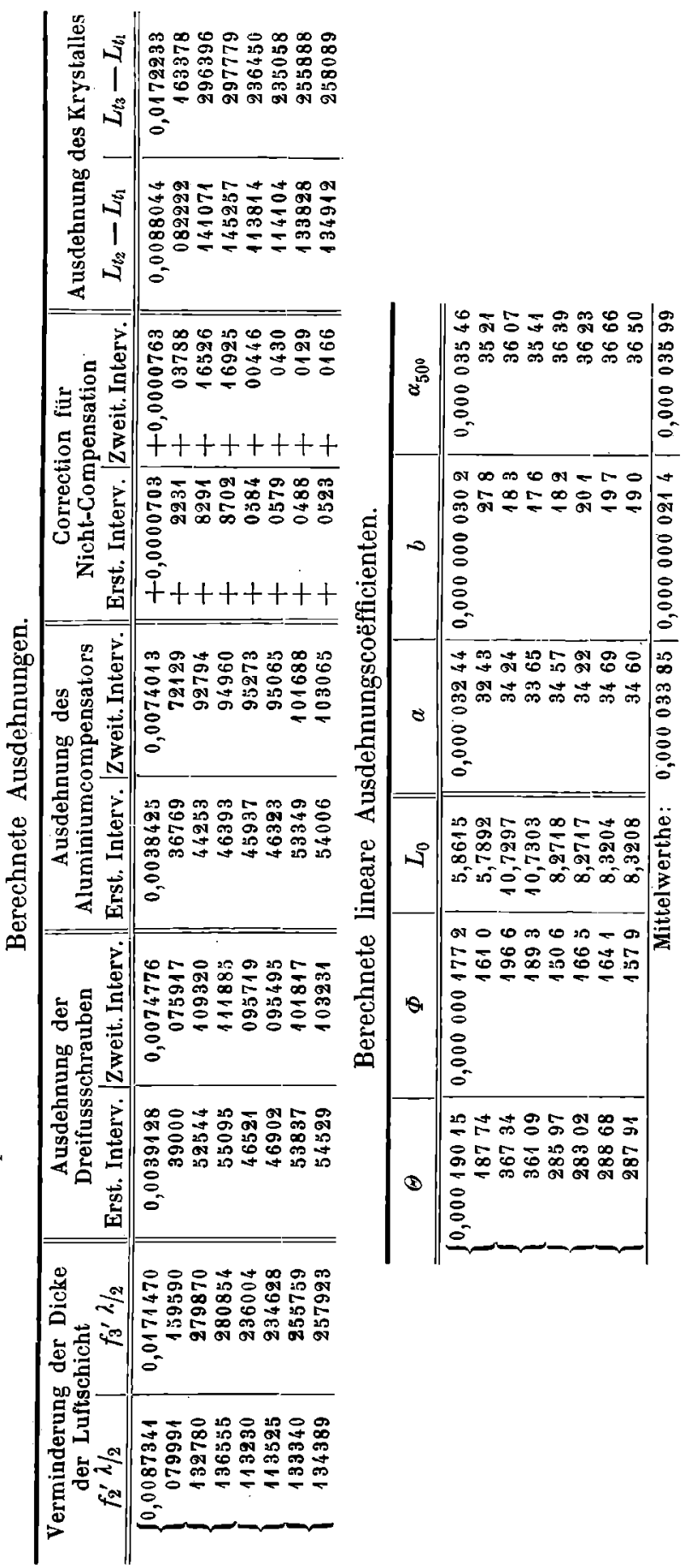


A. E. Tutton.
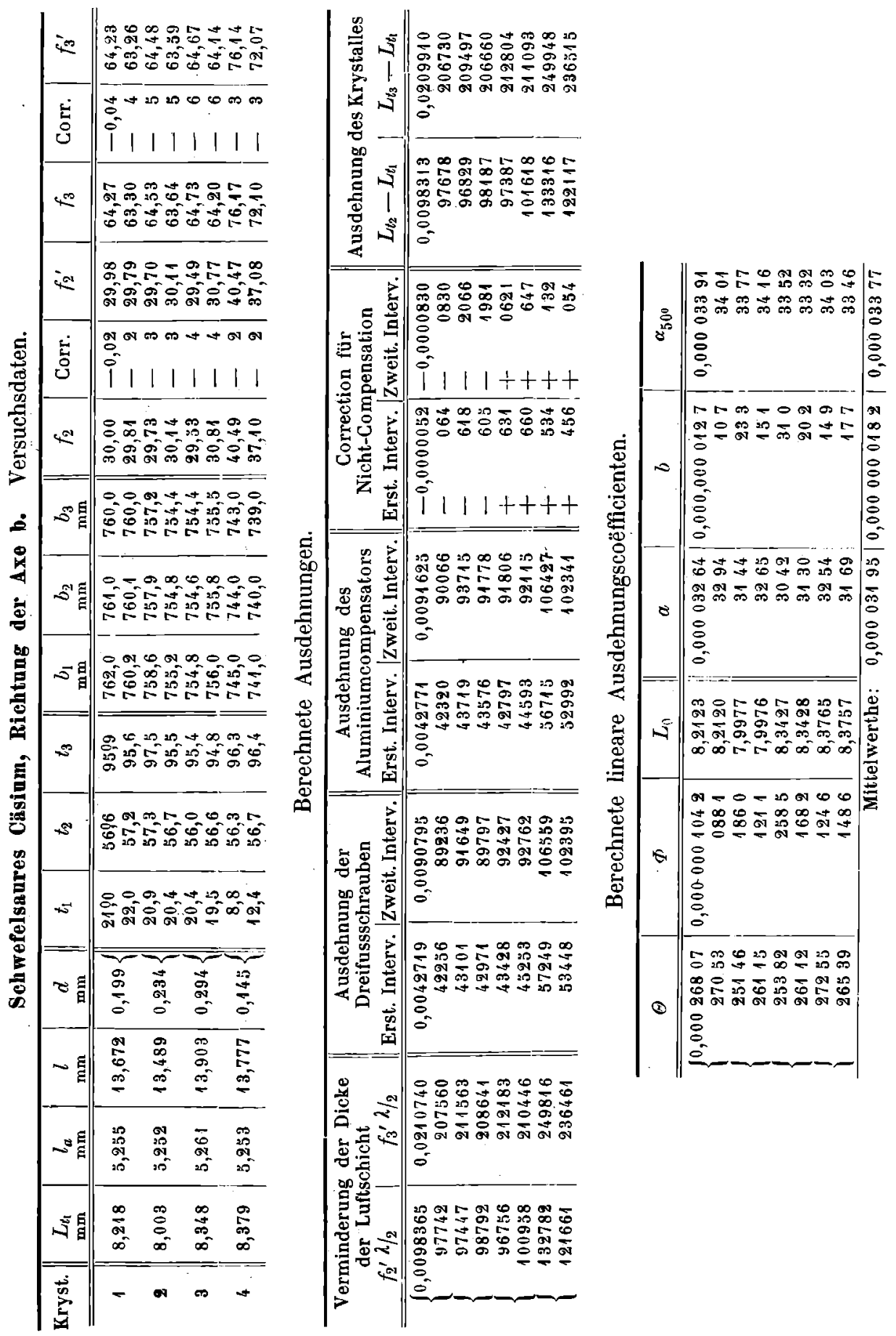

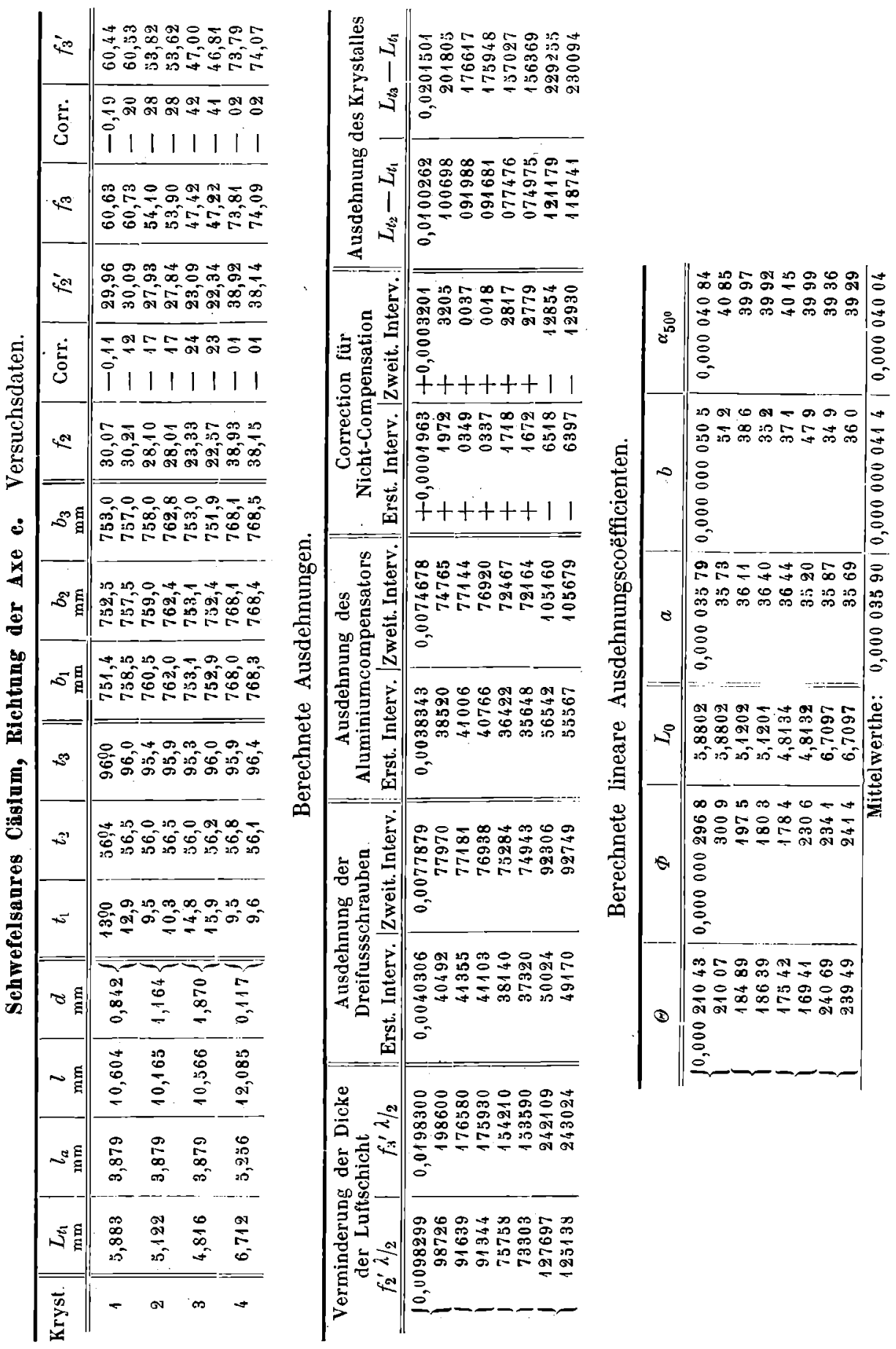


\section{Zusammenfassung der Resultate für die linearen Ausdehnungs- coëfficienten.}

In der folgenden Tabelle sind die Mittelwerthe der linearen Ausdehnungscoëfficienten zusammengestellt.

Mittlere lineare Ausdehnungscoëffleienten, $a+b t$, zwischen $0^{0}$ und to.

Schwefelsaures Kalium.

$$
\begin{aligned}
& \text { Richtung der Axe } a \quad 0,00003616+0,0000000144 t \\
& \text { - } \quad \text { - } \quad b \quad 0,00003225+0,0000000141 t \\
& \text { - } \quad-\quad c \quad 0,00003634+0,0000000413 t
\end{aligned}
$$

Schwefelsaures Rubidium.

$$
\begin{array}{ccccc}
\text { Richtung der Axe } & a & 0,00003637+0,0000000203 t \\
- & - & - & b & 0,00003214+0,0000000184 t \\
- & - & - & c & 0,00003463+0,0000000380 t
\end{array}
$$

Schwefelsaures Cäsium.

$$
\begin{aligned}
& \text { Richtung der Axe } a \quad 0,00003385+0,0000000214 t \\
& \text { - } \quad-\quad b \quad 0,00003195+0,0000000182 t \\
& \text { - } \quad-\quad c \quad 0,00003590+0,0000000414 t
\end{aligned}
$$
werden.

In verkürzter Weise werden jetzt die wahren Coëfficienten gegeben

Wahre lineare Ausdehnungscoëfficienten $\alpha$ bei $t^{n}$ oder mittlere Coëfficienten zwischen irgend zwei Temperaturen, deren Mitte $t$ ist, $\alpha=a+2 b t$.

$$
\begin{gathered}
K_{2} S O_{4} \\
\alpha_{a}=10^{-8}(3616+2,88 t) \\
\alpha_{b}=10^{-8}(3225+2,82 t) \\
\alpha_{c}=10^{-8}(3634+8,26 t) \\
R b_{2} S O_{4} \\
\alpha_{a}=10^{-8}(3637+4,06 t) \\
\alpha_{b}=10^{-8}(3214+3,68 t) \\
\alpha_{c}=10^{-8}(3463+7,60 t) \\
C s_{2} S O_{4} \\
\alpha_{a}=10^{-8}(3385+4,28 t) \\
\alpha_{b}=10^{-8}(3195+3,64 t) \\
\alpha_{c}=10^{-8}(3590+8,28 t)
\end{gathered}
$$

Eine Vergleichung der Ausdehnungscoëfficienten für analoge Richtungen in den drei Salzen wird in der folgenden Tabelle dargestellt. 


\section{Vergleichstabelle der linearen Ausdehnungscoëfficienten für die drei Salze.}

Die Constante $a$, der Ausdehnungscoëfficient bei $0^{0}$.

$\begin{array}{cccc}\text { Axenrichtung: } & \mathrm{K}_{2} \mathrm{SO}_{4} & \mathrm{Rb}_{2} \mathrm{SO}_{4} & \mathrm{Cs}_{2} \mathrm{SO}_{4} \\ a & 0,00003616 & 0,00003637 & 0,00003385 \\ b & 0,00003225 & 0,00003214 & 0,00003195 \\ c & 0,00003634 & 0,00003463 & 0,00003590 \\ \text { Summe } & 0,00010475 & 0,00010314 & 0,00010170\end{array}$

Die Constante $b$, die Hälfte des Increments.

$\begin{array}{cccc}\text { Axenrichtung: } & \mathrm{K}_{2} \mathrm{SO}_{4} & \mathrm{Rb}_{2} \mathrm{SO}_{4} & \mathrm{Cs}_{2} \mathrm{SO}_{4} \\ a & 0,0000000144 & 0,0000000203 & 0,0000000214 \\ b & 0,0000000141 & 0,0000000184 & 0,0000000182 \\ c & 0,0000000413 & 0,0000000380 & 0,0000000414 \\ \text { Summe } & 0,0000000698 & 0,0000000767 & 0,0000000810\end{array}$

\begin{tabular}{|c|c|c|c|}
\hline $\begin{array}{c}\text { Axenrichtung: } \\
a\end{array}$ & $\begin{array}{c}K_{2} \mathrm{SO}_{4} \\
0,00003760\end{array}$ & $\begin{array}{c}R b_{2} S O_{4} \\
0,00003840\end{array}$ & $\begin{array}{c}\mathrm{Cs}_{s_{2}} \mathrm{SO}_{4} \\
0,00003599\end{array}$ \\
\hline$b$ & 0,00003366 & 0,00003398 & 0,00003377 \\
\hline$c$ & 0,00004047 & 0,00003843 & 0,00004004 \\
\hline Summe & 0,00011173 & 0,00011081 & 0,00010980 \\
\hline
\end{tabular}

In der vorhergehenden Tabelle sind die Summen der Werthe für jede Constante für die drei Axenrichtungen jedes Salzes gegeben. Solche Summen repräsentiren, wie wohlbekannt ist, die Constanten der kubischen Ausdehnünscoëfficienten. Denn wenn man den Ausdruck für die Producte der Ausdehnungen in den drei Axenrichtungen untersucht, findet man, dass die einzigen Glieder, welche die vier letzten Decimalen berühren, die Summen der Constanten $a$ resp. $b$ sind.

In der nächsten Tabelle sind die so erhaltenen kubischen Ausdehnungscoëfficienten vergleichbar aufgestellt.

Kubische Ausdehnungscoëfficienten.

\begin{tabular}{|c|c|c|c|}
\hline & $a$ & $b$ & $\alpha_{50^{\circ}}$ \\
\hline & 0,00010475 & 0,0000000698 & 0,00011173 \\
\hline & 0,00010314 & 0,0000000767 Diff. 49 & 0,00011081 \\
\hline $\mathrm{SO}$ & $0,00010170^{1}$ & $0,0000000810^{011}$ & $0,00010980^{1}$ \\
\hline
\end{tabular}

Die mittleren kubischen Ausdehnungscoëfficienten der drei Salze zwischen $0^{0}$ und $t^{0}$ sind also die folgenden.

Für schwefelsaures Kalium:

$$
0,00010475+0,0000000698 t \text { oder } 10^{-8}(10475+6,98 t) \text {. }
$$


Für schwefelsaures Rubidium:

$$
0,00010314+0,0000000767 t \text { oder } 10^{-8}(10314+7,67 t) \text {. }
$$

Für schwefelsaures Cäsium:

$$
0,00010170+0,0000000810 t \text { oder } 10^{-8}(10170+8,10 t) \text {. }
$$

Die wahren kubischen Ausdehnungscoëfficienten $\alpha$, bei irgend einer Temperatur $t$ und auch die mittleren Coëfficienten zwischen irgend zwei

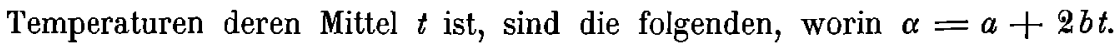

Für schwefelsaures Kalium:

$$
\alpha=0,00010475+0,0000001396 t \text { oder } 10^{-8}(10475+13,96 t) .
$$

Für schwefelsaures Rubidium:

$$
\alpha=0,00010314+0,0000001534 t \text { oder }^{2} 10^{-8}(10314+15,34 t) \text {. }
$$

Für schwefelsaures Cäsium:

$$
\alpha=0,00010170+0,0000001620 t \text { oder } 10^{-8}(10170+16,20 t) \text {. }
$$

\section{Erörterung der Resultate und Schlussfolgerungen.}

\section{Die kubische Ausdehnung.}

Das Hauptresultat der Untersuchung ist:

Die kubischen Ausdehnungscoefficienten der normalen Sulfate von Kalium, Rubidium und Cäsium zeigen ein Fortschreiten, welches dem Fortschreiten der Atomgewichte der drei respectiven Metalle entspricht. Dieses gilt von den beiden Constanten $a$ und $b$ des allgemeinen Ausdruckes für den kubischen Ausdehnungscoëfficienten.

Die Differenzen zwischen den Werthen der Constante $a$, des Coëfficienten bei $0^{0}$, für die drei Salze sind gering, nur $1 \frac{1}{2} \%$; dieses ist aber ein Betrag, welcher fünfmal so gross als der mögliche Beobachtungsfehler ist. Man sieht ferner :

Der Sinn des Fortschreitens der zwei Constanten ist entgegengesetzt; $a$, der Coëficient bei $0^{0}$, vermindert sich mit zunehmendem Atomgewichte des in dem Salze enthaltenen Metalles, während $b$, das Halbincrement pro Grad, zunimmt.

Die letztere Thatsache verursacht, dass die mit steigender Temperatur zunehmenden Coëfficienten sich einander nähern, bis sie bei drei besonderen Temperaturen zwischen $110^{\circ}$ und $170^{\circ}$ paarweise identisch werden; in der Nähe der zweiten dieser Temperaturen liegen die drei Werthe so dicht beisammen, dass die Diflerenz die Beobachtungsfehlergrenzen berührt. Für höhere Temperaturen als diejenigen, bei welchen Kreuzung vorkommt, divergiren die Werthe und zeigen einen entgegengesetzten Sinn des Fortschreitens. Dieses wird klar aus einer Tabelle hervorgehen, welche die Coëfficienten der kubischen Ausdehnung $a+2 b t$ für besondere Temperaturen bis $200^{\circ}$ darstellt. 
Kubische Ausdehnungscoëfficienten für Temperaturen von $0^{0}$ bis $200^{\circ}$.

\begin{tabular}{c||c|c|c|c|c|c|c|c}
\hline Salz & 00 & $50^{0}$ & 1000 & 1140 & $136^{0}$ & 1500 & $168^{0}$ & 2000 \\
\hline \hline$K_{2} S_{4}$ & $10^{-9} \cdot 10475$ & 11173 & 11871 & 12066 & 12373 & 12569 & 12820 & 13267 \\
$R b_{2} S O_{4}$ & $10^{-8} \cdot 10314$ & 11081 & 11848 & 12065 & 12400 & 12615 & 12891 & 13382 \\
$C s_{2} S O_{4}$ & $10^{-8} \cdot 10170$ & 10980 & 11790 & 12017 & 12373 & 12600 & 12891 & 13410
\end{tabular}

Dieses wird ferner klar mit Hülfe der graphischen Darstellung in beistehender Figur, in welcher als Abscisse die Temperaturen und als Ordinaten die kubischen Ausdehnungscoëficienten genommen worden sind. Die drei Linien convergiren von $0^{0}$ nach den Coincidenztemperaturen, und darüber

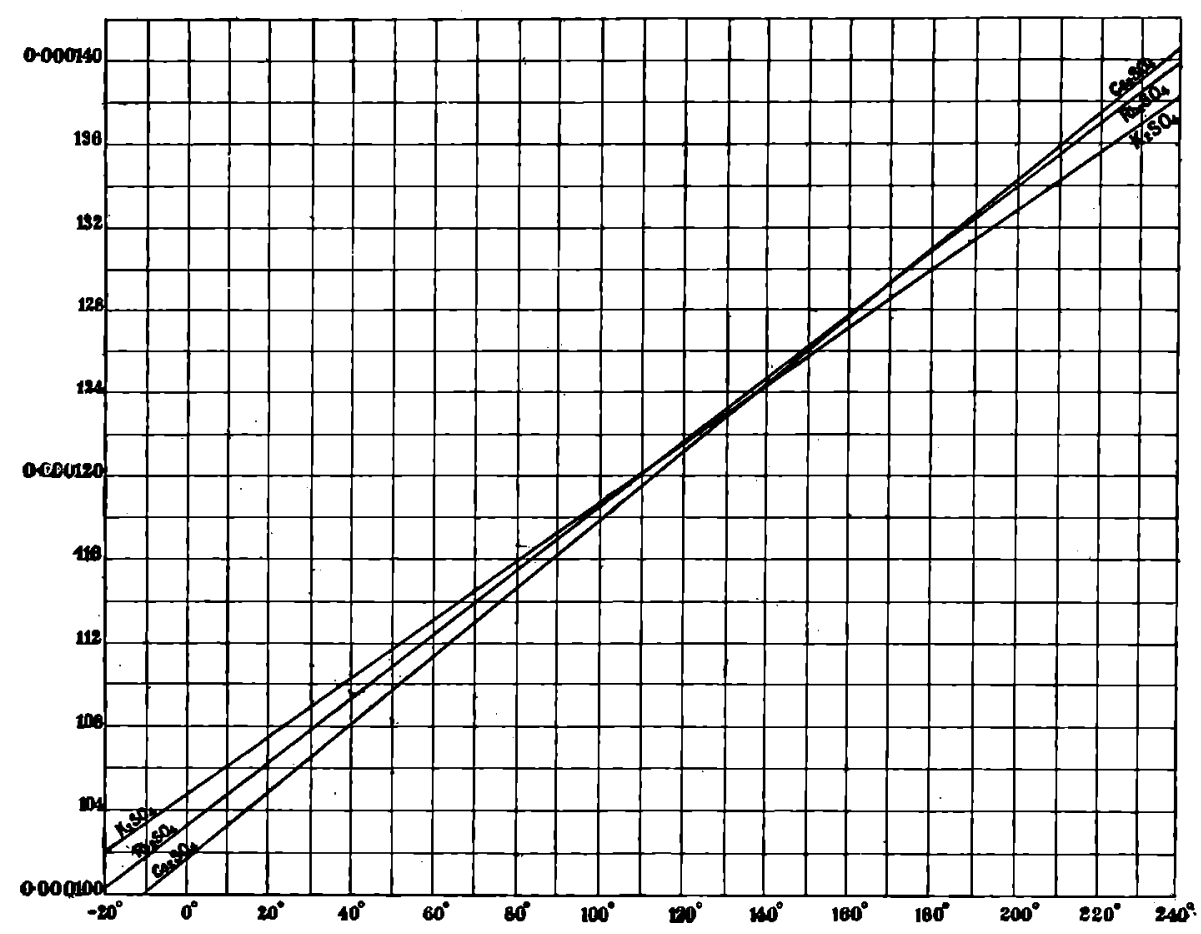

divergiren sie. Die relative Nähe der Linien an der Zone der Kreuzung, nebst der Thatsache, dass das Increment $2 b$ nicht so genau bestimmbar ist; wie der Coëfficient bei $0^{0}$, $a$, deuten an, dass vielleicht die drei Linien sich in einem und demselben Punkte kreuzen sollten, in der Nähe von $136^{\circ}$, anstatt paarweise bei $114^{\circ}, 136^{\prime \prime}$ und $168^{\circ}$. In der That ist bei $136^{\circ}$ der Werth für schwefelsaures Rubidium nur um ein Fünfhundertstel verschieden von den zwei identischen Werthen für die zwei anderen Salze. Also sind die drei Werthe in der Nähe dieser Temperatur innerhalb 
der Versuchsfehlergrenzen identisch. Ob die Werthe wirklich paarweise, oder alle drei bei einer specifischen Temperatur identisch werden, kann das Experiment nicht entscheiden. Da ein Fortschreiten nach dem Atomgewichte klar erkennbar ist, scheint es am wahrscheinlichsten, dass die Kreuzung bei einer einzigen Temperatur vor sich geht; andernfalls wäre die mittlere Stellung des Rubidiumsalzes zwischen $114^{\circ}$ und $168^{\circ}$ nicht erhalten.

Also kann man die folgende Schlussfolgerung hinzufügen:

Die kubischen $\Lambda$ usdehnungscoëficienten convergiren mit Steigerung der Temperatur gegen die Gleichheit, welche sie innerhalb der Versuchsfehlergrenzen bei $136^{\circ}$ erreichen. Bei höheren Temperaturen divergiren die Coëfficienten mit umgekehrtem Sinne des Fortschreitens, d. h. eine Zunahme des Atomgewichtes des Metalls wird jetzt von einer Zunahme des kubischen Ausdehnungscoëficienten begleitet, während bei der Convergenz eine Zunahme des Atomgewichtes von einer Verminderung des Coëfficienten begleitet wird.

\section{Die lineare Ausdehnung.}

Die folgenden Schlussfolgerungen sind aus den linearen Coëfficienten zu ziehen.

Die thermische Deformation besteht in einer Ausdehnung nach allen Richtungen.

Der Betrag der Ausdehnung ist relativ gross, mit den Ausdehnungen von Metallen verglichen, nämlich viermal so gross als die Ausdehnung des Platins und anderthalbmal so gross als diejenige von Aluminium.

Die Differenzen $z$ wischen den Beträgen der linearen Ausdehnung längs der drei Axenrichtungen irgend eines Salzes sind gering, hüchstens $12 \%$ der gesammten Ausdehnung. Noch geringer sind aber die Differenzen zwischen den Werthen für dieselbe Richtung der drei Salze; im Falle der Axenrichtung $b$ ist die Differenz nur 1\%, und im Falle der Axe $a$, wo die grösste Differenz vorkommt, ist sie nur $6 \%$ der Gesammtausdehnung.

Das Increment des Ausdehnungscoëfficienten pro Temperaturgrad ist circa zweimal so gross für die Richtung der Axe $c$ jedes Salzes, als für die zwei anderen Axenrichtungen, für welche die Incremente beinahe identisch sind.

Die letztere Thatsache stimmt auffallend mit der früher mitgetheilten optischen Thatsache (diese Zeitschr. 24, 75, \& 14) dass die Veränderung des Refractionsvermögens mit Temperatursteigerung beträchtlich grösser für die Richtung $c$ ist, als für die Richtungen $a$ und $b$, längs welcher die Veränderung approximativ dieselbe ist. 
Der Ausdehnungsbetrag längs der Axenrichtung $b$ ist praktisch identisch für alle drei Sulfate, also ist die Vertauschung der drei Metalle ohne Einfluss auf das thermische Verhalten längs dieser, der makrodiagonalen Axe der Krystalle. Ausserdem dehnen sich die Krystalle aller drei Salze am wenigsten längs dieser Richtung aus, welche daher diejenige der minimalen Axe des thermischen Ellipsoids ist.

Diese beiden Thatsachen sind zweifellos wichtig in Hinsicht auf den Bau des Moleküls. Es scheint, als wenn die metallischen Atome oder ihre Bewegungskreise entfernt von der Gegend dieser Axe sind. Die Wichtigkeit ist um so grösser, da Verfasser früher gezeigt hat (diese Zeitschr. 27, 283), dass die ganze Arbeit über die Sulfate und Doppelsulfate mit der Voraussetzung übereinstimmt, dass die Structureinheiten der Krystalle der Sulfate die einfachen chemischen Molekeln sind, eine Schlussfolgerung, welche durch die Arbeit von Fock (Ber. d. deutsch. chem. Ges. 1895, 28, 2734 und diese Zeitschr. 1895, 25, 566) über die Löslichkeit von Mischkrystallen gestützt ist.

Die Kleinheit der Ausdehnungsdifferenz längs irgend einer besonderen Richtung in den Krystallen, welche durch die Ersetzung eines Alkalimetalles durch ein anderes hervorgebracht wird, verglichen mit den grösseren Ausdehnungsdifferenzen, welche in den drei Axenrichtungen irgend eines Salzes sich zeigen, nebst der Thatsache, dass die Vertauschung der Metalle von beträchtlichen Modificationen dieser letzteren relativen Ausdehnungen für zwei der Axenrichtungen $a$ und $c$ begleitet wird, verursacht, dass die Goëfficienten für diese Axenrichtungen gar kein Fortschreiten nach dem Atomgewichte zeigen können.

Es mag von Interesse sein, die wahrscheinliche Grösse der linearen Veränderung zu betrachten, welche durch Ersetzung eines Metalles durch ein anderes hervorgebracht würde, wenn keine solche Störung der Richtungsbeziehungen vorkäme. Die Differenz zwischen den kubischen Ausdehnungscoëfficienten von Kalium- und Rubidiumsulfat ist 0,00000161 und von Rubidium- und Cüsiumsulfat 0,00000144. Das Mittel ist 0,0000015, und die linearen Differenzen können ein Drittel dieses Werthes, 0,0000005, sein. Selbst wenn die Richtung $\operatorname{der}$ Axe $b$ absolut unberührt durch Metallersetzung wäre, künnten die linearen Differenzen für die Axenrichtungen $a$ und $c$ nicht 0,0000008 überschreiten. Nun beträgt aber die hauptsächliche Richtungsstörung, in welcher das Rubidiumsalz eine Umkehrung der Richtungen der maximalen und mittleren thermischen Axen zeigt, mit dem Kaliumsalz verglichen, mehr als das Doppelte jenes Betrages, nämlich 0,0000017 . Also ist es klar, dass eine progressive Veränderung des maxi- 
malen müglichen Betrages vollständig maskirt sein würde durch die grössere Richtungsveränderung.

Wenn. man die linearen Ausdehnungscoëfficienten für $0^{0}$ studirt, bemerkt man:

Die Ausdehnung längs der Axe $e$ ist die grüsste bei dem Kalium- und dem Cäsiumsalze. Wie früher angegeben, ist bei allen drei Salzen das Increment für diese Richtung grüsser, als für irgend eine andere Richtung. $\Lambda$ ber im Falle des Rubidiumsalzes ist die $\Lambda$ usdehnung am grössten für die Axenrichtung $a$, während diejenige für $c$ die mittlere ist. Die Incremente pro Grad aber bleiben von derselben Ordnung, wie für die anderen beiden Salze.

Von Interesse ist es, dass die Richtungen der maximalen thermischen Ausdehnung mit derjenigen der ersten Mittellinie der optischen Axenwinkel aller drei Salze übereinstimmen, welche die Axe $c$ bei dem Kalium- und dem Cäsiumsalze und die $\Lambda$ xe $a$ bei dem Rubidiumsalze ist.

Um diesen. Parallelismus weiter zu verfolgen, ist eine Tabelle beigegeben, in welcher die Werthe von $\alpha=a+2 b t$ für $0^{\circ}, 50^{\circ}$ und $100^{\circ} \mathrm{zu}$ sammengestellt sind, nebst den Richtungen der Axen der optischen Indicatrix und dem Zeichen der Doppelbrechung.

Vergleichung der linearen Ausdehnungscoëfficienten mit der optischen Indicatrix.

\begin{tabular}{|c|c|c|c|c|c|c|}
\hline Salz & $\begin{array}{c}\text { Zeichen } \\
\text { der Doppel- } \\
\text { brechung }\end{array}$ & $\begin{array}{l}\text { Krystallo- } \\
\text { graphische } \\
\text { Axe }\end{array}$ & $\begin{array}{c}\text { Richtung der } \\
\text { optischen Indicatrix }\end{array}$ & $\alpha_{00}$ & $\alpha_{500}$ & $\alpha_{1000}$ \\
\hline $\mathrm{K}_{2} \mathrm{SO}_{4}$ & positiv & $\begin{array}{l}a \\
b \\
c\end{array}$ & $\begin{array}{l}\text { Mittlere Axe } \\
\text { Zweite Mittellinie } \\
\text { Erste Mittellinie }\end{array}$ & \begin{tabular}{|r|}
$10^{-8} \cdot 3616$ \\
3225 \\
3634 \\
\end{tabular} & $\begin{array}{l}3760 \\
3366 \\
4047\end{array}$ & $\begin{array}{l}3904 \\
3507 \\
4460\end{array}$ \\
\hline $\mathrm{Rb}_{2} \mathrm{SO}_{4}$ & $\begin{array}{c}\text { sehr sehwach } \\
\text { positiv }\end{array}$ & $\begin{array}{l}a \\
b \\
c\end{array}$ & $\begin{array}{l}\text { Erste Mittellinie } \\
\text { Zweite Mittellinie } \\
\text { Mittlere Axe }\end{array}$ & $\begin{array}{l}3637 \\
3214 \\
3463\end{array}$ & $\begin{array}{l}3840 \\
3398 \\
3843\end{array}$ & $\begin{array}{l}4043 \\
3582 \\
4223\end{array}$ \\
\hline $\mathrm{Cs}_{2} \mathrm{SO}_{4}$ & negativ & $\begin{array}{l}a \\
b \\
c\end{array}$ & $\begin{array}{l}\text { Zweite Mittellinie } \\
\text { Mittlere Axe } \\
\text { Erste Mittellinie }\end{array}$ & $\begin{array}{l}3385 \\
3195 \\
3590\end{array}$ & $\begin{array}{l}3599 \\
3377 \\
4004\end{array}$ & $\begin{array}{l}3813 \\
3559 \\
4418\end{array}$ \\
\hline
\end{tabular}

Es geht aus der Tabelle klar hervor, dass die Beziehungen der linearen Ausdehnungscoëfficienten bei $0^{0}$ noch gültig sind bei höheren Temperaturen in den Fällen des Kalium- und Cäsiumsalzes. Eine ähnliche Thatsache ergab sich auch für die optischen Eigenschaften als gültig. Im Falle des Rubidiumsalzes kommt jedoch eine merkwürdige Veränderung vor: wegen des grüsseren Incrementes längs $c$ vermindert sich der Ueberschuss der Aus- 
dehnung längs $a$, bis bei $0^{0}$ die Ausdehnungsbeträgre längs dieser zwei Axenrichtungen gleich werden; d. h. bei $50^{0}$ verhalten sich die Krystalle des schwefelsauren Rubidiums wie einaxige. Dies ist um so interessanter, als bei dieser Temperatur sie sich auch optisch einaxig verhalten (l. c. S. 57): Da eine beträchtliche Dispersion der Axen stattfindet, sind die Temperaturen für die Erreichung der Einaxigkeit etwas verschieden, nämlich $\mathbf{4 2}^{0}$ für rothes Lithiumlicht, $44^{\circ}$ für $C$-Wasserstofflicht, $48^{\circ}$ für Natriumlicht, $52^{0}$ für grünes Thalliumlicht und $58^{0}$ für grünlich-blaues $F$-Wasserstofllicht, also im Mittel 50\%. Aber die zwei Rotationsellipsoide für die beiden Eigenschaften sind nicht in ähnlicher Weise orientirt; die Hauptaxe für die oplische Eigenschaft ist $a$, während sie für die thermische Eigenschaft $b$ ist.

Bei $100^{\circ}$ und noch höher ist die maximale thermische Axe identisch mit $c$ für alle drei Salze, weil die fortlaufende schnellere Veränderung des $c$-Werthes die frühere exceptionelle Ordnung bei dem Rubidiumsalz umgekehrt hat. Bei $180^{\circ}$ ist auch die erste optische Mittellinie dieses Salzes von $a$ auf $c$ übergegangen, so dass bei dieser Temperatur $c$ die erste Mittellinie für alle drei Salze ist. Also gilt bei hüheren Temperaturen durchweg die Regel, dass die Richtung der maximalen thermischen Ausdehnung auch die Richtung der ersten optischen Mittellinie ist.

Diese Thatsachen kann man wie folgt zusammenfassen:

Bei höheren Temperaturen gelten dieselben thermischen und optischen Beziehungen, wie bei Temperaturen unter $50^{\circ}$ in den Fällen des Kalium- und Cäsiumsalzes. Da aber das Ausdehnungsincrement pro Grad längs der Axe $c$ viel grösser als für andere Richtungen ist, so wird die exceptionelle mittlere $\Lambda$ usdehnung längs $c$ für das Rubidiumsalz schnell zur Gleichheit, bei $50^{\circ}$, mit der $\Lambda$ usdehnung längs $a$ gebracht; über dieser Temperatur wird $c$ die maximale thermische Axe für das Rubidiumsalz, wie sie es auch für die anderen zwei Sulfate ist. Bei 50" sind demnach die Krystalle von schwefelsaurem Rubidium thermisch einaxig. Bei Temperaturen, welche für verschiedene Wellenlängen des Lichtes $10^{\circ}$ auf jeder Seite von $50^{\circ}$ variiren, sind sie auch optisch einaxig. Die thermischen und optischen Umdrehungsellipsoide aber sind nicht identisch orientirt; die Axe des ersteren ist $b$ und des letzteren $a$. Ferner ist die Richtungsveränderung der maximalen thermischen $\Lambda x e$ des schwefelsauren Rubidiums von a zu $c$ von der optischen Veränderung der ersten Mittellinie von $a$ zu $c$ bei $180^{\circ}$ begleitet. Also entspricht die erste optische Mittellinie, wie bei niedrigeren Temperaturen, der maximalen thermischen Axe für alle drei Salze.

Dieser Parallelismus zwischen den linearen thermischen Ausdehnungscoëfficienten und den optischen Constanten ist von Wichtigkeit, da die letz- 
teren Constanten, welche, den ersteren entgegengesetzt, zwischen den drei Salzen viel grössere Differenzen als die Richtungsdifferenzen für irgend ein Salz zeigen, ein deutliches Fortschreiten mit dem Atomgewichte des Metalles zeigen. Um dieses klar zu machen, sind die Brechungsindices für Natriumlicht aus der früheren Mittheilung (diese Zeitschr. 24, 59) hier gegeben.

\section{Brechungsexponenten der Sulfate für Natriumlicht.}

$\begin{array}{cccc}\text { Axenrichtung: } & \mathrm{K}_{2} \mathrm{SO}_{4} & \mathrm{Rb}_{2} \mathrm{SO}_{4} & \mathrm{Cs}_{2} \mathrm{SO}_{4} \\ a & 1,4947 & 1,5144 & 1,5662 \\ b & 1,4935 & 1,5131 & 1,5644 \\ c & 1,4973 & 1,5133 & 1,5598\end{array}$

Es wird endlich interessant sein, die Resultate, welche jetzt mit der empfindlichen Interferenzmethode erhalten worden sind, mit denjenigen zu vergleichen, welche früher (1. c. S. 23) aus der Verbindung von Dichtigkeitsbestimmungen bei den gewöhnlichen und höheren Temperaturen mit Messungen der morphologischen Winkel bei jenen Temperaturen erhalten worden waren.

Die gefundenen Werthe für die gesammte kubische Ausdehnung für $40^{\circ}$ (zwischen $20^{\circ}$ und $60^{\circ}$ ) waren:

$$
\begin{array}{ll}
\mathrm{K}_{2} \mathrm{SO}_{4} & 0,0053 \\
\mathrm{Rb}_{2} \mathrm{SO}_{4} & 0,0052 \\
\mathrm{Cs}_{2} \mathrm{SO}_{4} & 0,0051
\end{array}
$$

Also ergab sich eine Verminderung der Ausdehnung mit einer Zunahme des Atomgewichtes des Metalles, ein Resultat, welches mit den genaueren jetzt mitgetheilten Bestimmungen übereinstimmt. Die Werthe für die drei Salze waren so nahe identisch, dass sie als identisch in Rücksicht auf die Bestimmungsmethode genommen wurden, für den Zweck, die linearen Coëfficienten mit Hülfe der Winkelveränderungen zwischen denselben Temperaturen zu berechnen. Die folgenden Werthe wurden für die linearen Ausdehnungscoëfficienten für gesammte $100^{\circ}$ gegeben $(\lambda=$ das $\alpha$ dieser Mittheilung):

$$
\begin{aligned}
& \lambda_{a}=0,00437 \\
& \lambda_{b}=0,00385 \\
& \lambda_{c}=0,00479
\end{aligned}
$$

Es wurde daher geschlossen, dass »die Krystalle der drei Salze sich durch Erhitzen am meisten längs der Verticalaxe $c$ ausdehnen und am wenigsten in der Richtung der Makrodiagonalaxe $b_{\ll}$. Dieses ist in der That richtig, mit Ausnahme des Falles von $\lambda_{a}$ und $\lambda_{c}$ des Rubidiumsalzes unter $50^{\circ}$; diese Ausnahme konnte nach jener Methode nicht entdeckt werden, weil bei $60^{\circ}$, der Temperatur der höheren Dichtigkeitsbestimmung, die gefundene Regel wirklich wahr ist. 
Die wahren Werthe für die gesammte Ausdehnung, zum Beispiel des schwefelsauren Kaliums, für das Intervall von $100^{\circ}$ zwischen $20^{\circ}$ und $120^{\circ}$, aus der Formel $\alpha=100^{\circ}\left[a+2 b\left(\frac{20^{0}+120^{0}}{2}\right)\right]$ berechnet, sind :

$$
\begin{aligned}
& \alpha_{a}=0,003818 \\
& \alpha_{b}=0,003422 \\
& \alpha_{c}=0,004212
\end{aligned}
$$

Die Differenzen zwischen diesen und den früheren Werthen geben eine Idee der relativen Genauigkeit der Methoden. Es ist befriedigend, dass die Ordnung dieselbe ist, und sie bestätigt die Schlussfolgerungen aus den Messungen der morphologischen Winkel bei verschiedenen Temperaturen, welche mit Hülfe des Fuess'schen Goniometers Nr. la ausgeführt worden sind.

Spring hat die Ausdehnung der Krystalle der Sulfate von Kalium und Rubidium vermittelst einer Gewichtsthermometermethode untersucht (Bull. de l'Acad. de Belgique 1882, 197 und Ber. d. d. chem. Ges. 15, 1940). Als Flüssigkeit wurde Olivenöl angewandt, und die Dichtigkeitsbestimmungen wurden bei $100^{\circ}$ ausgeführt. Der erhaltene Werth für die kubische Ausdehnung des schwefelsauren Kaliums für $100^{\circ}$ war 0,0126 und für Rubidiumsulfat 0,0111 . Der letztere Werth ist auffallend nahe der Wahrheit, welche nach dem jetzt mitgetheilten Resultate 0,01108 ist. Aber die Unzuverlässigkeit der Methode, richtige Differenzen zwischen den Werthen für verschiedene Salze zu liefern, wird klar durch die Thatsache bewiesen, dass die angedeutete Differenz zwisehen den Werthen für die Kalium- und Rubidiumsalze, nämlich 0,00150 , siebenzehnmal so gross als die wahre Differenz $(0,01117-0,01108=0,00009)$ ist, welche sich aus den obigen Beobachtungen ergab.

\section{Zusammenfassung der Schlussfolgerungen.}

Die Hauptresultate dieser Untersuchung sind die folgenden:

1. Die kubischen Ausdehnungscoëfficienten der orthorhombischen Krystalle der normalen Sulfate von Kalium, Rubidium und Cäsium zeigen ein Fortschreiten, welches dem Fortschreiten der Atongewichte der drei respectiven Metalle entspricht. Dieses gilt von den beiden Constanten $a$ und $b$ des allgemeinen Ausdrucks für den kubischen Ausdehnungscoëfficienten, $\alpha=a+2 b t$, für irgend eine Temperatur $t$.

2. Der Sinn des Fortschreitens der beiden Constanten ist entgegengesetzt; $a$, der Coëfficient bei $0^{0}$, vermindert sich mit zunehmendem Atomgewichte des in dem Salze enthaltenen Metalles, während $b$, das halbe Increment pro Grad, zunimmt.

3. Folglich convergiren die kubischen Ausdehnungscoëfficienten mit Steigerung der Temperatur gegen die Gleichheit, welche sie, innerhalb der 
Versuchsfehlergrenzen, bei $136^{0}$ erreichen. Bei höheren Temperaturen divergiren die Coëfficienten mit umgekehrtem Sinne des Fortschreitens; d. h. eine Zunahme des Atomgewichtes des Metalles wird jetzt von einer $\mathrm{Zu}$ nahme des kubischen Ausdehnungscoëfficienten begleitet, während bei der Convergenz eine Zunahme des Atomgewichtes von einer Verminderung des Coëfficienten begleitet wird.

4. Die thermische Deformation besteht in einer Ausdehnung nach allen Richtungen.

๖. Die Differenzen zwischen den linearen Ausdehnungscoëfficienten für die drei krystallographischen Axenrichtungen irgend eines Salzes, obgleich sie nur bis 12\% der gesammten Ausdehnung betragen, sind viel grösser, als die Differenzen zwischen den Werthen für dieselbe Richtung der drei Salze.

6. Die letztere Thatsache nebst der anderen, dass die Ersetzung eines Metalles durch ein anderes mit beträchtlichen Modificationen des Verhältnisses zwischen zweien der drei Werthe für das Ausgangssalz begleitet wird, nämlich derjenigen, welche den Axertrichtungen $a$ und $c$ entsprechen, verursacht, dass die Coëfficienten für irgend eine von diesen beiden Richtungen der drei Salze gar kein Fortschreiten nach dem Atomgewichte zeigen können.

7. Die Ausdehnung längs der Axenrichtung $b$ ist approximativ identisch für alle drei Salze. Also ist die Metallvertauschung ohne Einfluss auf die thermischen Beträge längs der makrodiagonalen Krystallaxe. Diese ist auch die Richtung minimaler Ausdehnung für jedes Salz.

8. Das Increment des Ausdehnungscoëfficienten pro Temperaturgrad ist circa zweimal so gross für die Richtung der Axe $c$ jedes Salzes, als für die beiden anderen Axenrichtungen, für welche die Incremente beinahe identisch sind. Dieses ist analog dem optischen Betrage, denn das Refractionsvermögen verändert sich bei Steigerung der Temperatur viel mehr längs der Richtung $c$, als längs der zwei anderen $\Lambda$ xenrichtungen, für welche die geringeren Veränderungen beinahe gleich sind.

9. Bei Temperaturen unter $5^{0}$ ist die Ausdehnung am grössten längs der Axenrichtung $c$ bei dem Kalium- und Cäsiumsalze, längs der $\Lambda$ xenrichtung $a$ aber im Falle des Rubidiumsalzes. Eine ähnliche Umkehrung der Richtung der ersten Mittellinie, der maximalen Axe der optischen Indicatrix, von $c \mathrm{zu} a$, kommt im Falle des Rubidiumsulfates vor. Also ist die maximale thermische Axe identisch in allen drei Salzen mit der ersten optischen Mittellinie.

10. Bei höheren Temperaturen gelten noch dieselben thermischen und optischen Beziehungen bei dem Kalium- und Cäsiumsalze. Aber wegen des grösseren Incrementes längs der Axe $c$ wird die exceptionelle mittlere Ausdehnung längs $e$ für Rubidiumsulfat zur Gleichheit bei $50^{0}$ mit der Ausdehnung längs a gebracht; über $50^{\circ}$ ist $c$ die maximale thermische Axe 
für alle drei Salze. Bei วั0" sind demnach die Krystalle von schwefelsaurem Rubidium thermisch einaxig. Bei Temperaturen von $42^{\circ}$ bis $58^{\circ}$, je nach der Wellenlänge des Lichtes, also im Mittel $50^{\circ}$, sind sie auch optisch einaxig. Die thermischen und optischen Umdrehungsellipsoide sind aber nicht identisch orientirt; deren. Axen sind $b$ resp. $a$. Ueber $180^{\circ}$ entspricht wieder die erste optische Mittellinie der maximalen thermischen $\Lambda$ xe für alle drei Salze, denn die erste Mittellinie des Rubidiumsalzes geht bei dieser Temperatur von $a$ auf $c$ über.

11. Ein Parallelismus ist also zwischen der linearen Ausdehnung und den optischen Eigenschaften erwiesen worden, und dieser deutet an, dass eine ähnliche Wirkung in Rücksicht auf Atomgewichte im Falle der ersteren wie im Falle der letzteren vorliegt, und dass diese Wirkung noch mehr bemerkbar im Falle der ersteren sein würde, wenn sie nicht von der grösseren im $\S 6$ erwähnten Wirkung maskirt wäre.

12. Diejenigen thermischen Deformationsconstanten, welche am besten die Wirkung der Ersetzung eines Metalles durch ein anderes zeigen, sind folglich die kubischen Ausdehnungscoëfficienten und ihre Incremente; und für diese ist ein Fortschreiten nach dem Atomgewichte des Metalles bewiesen worden. Ausserdem ist gezeigt worden, dass die linearen Coëfficienten und ihre Incremente Variationen darbieten, welche eine auffallende Analogie mit denjenigen der optischen Constanten zeigen; und für die letzteren ist ein deutliches Fortschreiten nach dem Atomgewichte der Metalle gefunden worden, indem die Werthe für die drei Salze viel weiter von einander entfernt sind und folglich durch die Modification der bei den thermischen Constanten so viel wichtigeren Richtungsbeziehungen gar nicht gestört werden.

Jie Hauptschlussfolgerung dieser Untersuchung ist daher die folgende:

Die thermischen Deformationsconstanten der Krystalle der normalen Sulfate von Kalium, Rubidium und Cäsium bieten Variationen dar, welche, gemeinschaftlich mit den früher untersuchten morphologischen, optischen und anderen physikalischen Eigenschaften, dem Sinne des Fortschreitens der Atomgewichte der in den Salzen enthaltenen Metalle folgen. 